\title{
Diversidad de plantas Vasculares del VAlle Antinaco-Los Colorados, La Rioja, Argentina
}

\author{
OMAR VARELA1,2, MARÍA F. PARRADO ${ }^{3}$ y SEBASTIÁN E. BUEDO ${ }^{1}$
}

\begin{abstract}
Summary: Vascular plant diversity of the valley Antinaco-Los Colorados, La Rioja Argentina. The Antinaco-Los Colorados Valley is an extensive plain of the Monte ecoregion in the center-west of La Rioja (Argentina). This study provides a comprehensive inventory of the diversity of vascular plants growing spontaneously in the valley. A total of 264 species distributed in 57 families and 181 genera were recorded in four types of vegetation during 90 field trips. Eighteen species are new records for the province. The most diverse families were Asteraceae (35 spp., 13\%), Poaceae (32 spp., 12\%), Fabaceae (19 spp., 7\%), Solanaceae (19 spp., 7\%) and Cactaceae (16 spp., 6\%). The best represented genera were Tillandsia (10 spp.), Solanum (6 spp.), Atriplex (5 spp.), Lycium (5 spp.) and Prosopis (5 spp.). The 13\% of species (35) are introduced, mostly belong to families Poaceae (8 spp.), Asteraceae (5 spp.), Brassicaceae (5 spp.) and Chenopodiaceae (5 spp.). The valley includes 84 endemisms of level National, Regional and Provincial, $41 \%$ of which belongs to the family Cactaceae (13 spp.), Asteraceae (9 spp.), Solanaceae (7 spp.), Chenopodiaceae (6 spp.) and Euphorbiaceae (6 spp.). The dominant forms of life were the Phanerophytes (28\%), Therophytes (28\%) and Hemicryptophytes $(21 \%)$. The valley concentrates approximately $17 \%$ of the diversity of vascular plants known for La Rioja.
\end{abstract}

Key words: Endemism, Vascular Flora, floristic inventory, life-forms, Monte Desert, Vegetation.

\begin{abstract}
Resumen: El valle Antinaco-Los Colorados es una extensa planicie de la ecorregión del Monte en el centro-oeste de La Rioja (Argentina). Este estudio proporciona un inventario exhaustivo de la diversidad de plantas vasculares que crecen espontáneamente en el valle. En 90 viajes de campo se registraron 264 especies de 57 familias y 181 géneros en 4 tipos de vegetación. Se mencionan 18 citas nuevas para la provincia. Las cinco familias más diversas fueron Asteraceae (35 spp., 13\%), Poaceae (32 spp., $12 \%)$, Fabaceae (19 spp., 7\%), Solanaceae (19 spp., 7\%) y Cactaceaae (16 spp., 6\%). Los géneros mejor representados fueron Tillandsia (10 spp.), Solanum (6 spp.), Atriplex (5 spp.), Lycium (5 spp.) y Prosopis (5 spp.). El 13\% de las especies (35) son introducidas, principalmente de las familias Poaceae (7 spp.), Asteraceae (5 spp.), Brassicaceae (5 spp.) y Chenopodiaceae (5 spp.). El valle incluye 84 endemismos de nivel nacional, regional y provincial, y en su mayoría (41\%) corresponden a Cactaceae (13 spp.), Asteraceae (9 spp.), Solanaceae (7 spp.), Chenopodiaceae (6 spp.) y Euphorbiaceae (6 spp.). Las formas de vida dominantes fueron los Fanerófitos (29\%), Terófitos (28\%) y Hemicriptófitos (21\%). El valle concentra aproximadamente el $17 \%$ de la diversidad de plantas vasculares conocida para La Rioja.
\end{abstract}

Palabras clave: Flora vascular, Catálogo florístico, endemismos, formas de vida, Monte, Vegetación.

\section{INTRODUCCIÓN}

Las regiones áridas, particularmente los desiertos y semidesiertos cálidos, son unidades geográficas

1 Instituto de Ecología, Fundación Miguel Lillo (FML), Miguel Lillo 251, San Miguel de Tucumán, CP 4000, Argentina. E-mail: omarvarela1@gmail.com

${ }^{2}$ Instituto de Ambientes de Montañas y Regiones Áridas (IAMRA), Universidad Nacional de Chilecito (UNdeC), La Rioja, Argentina.

${ }^{3}$ Herbario Fanerogámico (FML). sometidas a condiciones ambientales extremas (Noy-Meir, 1973). Se caracterizan por las escasas precipitaciones anuales $(\leq 250 \mathrm{~mm})$, la elevada evapotranspiración y por un índice de aridez inferior a 0,20 (FAO, 2007). Otros rasgos peculiares incluyen la alta irregularidad espacial y temporal de las precipitaciones, la elevada insolación y amplitud térmica, la alta frecuencia e intensidad de los vientos, así como el escaso desarrollo de los suelos y la baja productividad primaria (Noy-Meir, 1973; FAO, 2007). Estas características determinan que las zonas áridas sean extremadamente frágiles 
y vulnerables a la degradación y desertificación (MEA, 2005a).

En general las zonas secas han sido consideradas áreas de diversidad biológica muy limitada, por lo que han recibido poca atención en los programas de conservación (Moreno-Calles \& Casas, 2008). Sin embargo, actualmente se conoce que los desiertos albergan diversidad biológica de importancia en términos de adaptaciones y usos para el hombre (Darkoh, 2003), e incluyen algunas de las especies más amenazadas del mundo (Durant et al., 2012).

Debido a que la diversidad específica de las zonas áridas es menor en comparación con las zonas húmedas, la pérdida de especies en esas zonas tiene un impacto relativamente más alto, más aún si se considera la elevada proporción de endemismos que representan (McNeely, 2003). En este sentido, las zonas áridas y semiáridas de América Latina y el Caribe, constituyen importantes reservorios de endemismos y de especies catalogadas de rareza biogeográfica, de hábitat y demográfica (Halffter \& Ezcurra, 1992; Simonetti, 1996).

En Argentina, una de las regiones biogeográficas de características áridas con mayor extensión es el Monte $\left(460.000 \mathrm{~km}^{2}\right)$. Esta región se extiende en forma de faja al este de la cordillera de Los Andes, desde los $24^{\circ} 35^{\prime} \mathrm{S}$ (Jujuy y Salta) hasta los $44^{\circ} 20^{\prime} \mathrm{S}$ (Chubut), y desde los $2.500 \mathrm{msm}$ en Los Andes hasta la costa atlántica (Abraham et al., 2009). En esta región, se reconocen distintos tipos de vegetación (Morello, 1958). El tipo de vegetación predominante o de mayor extensión es el matorral o estepa de arbustos esparcidos (en adelante "estepa típica"), conformada por especies micrófilas, áfilas, espinosas y resinosas. Florísticamente, se caracteriza por el predominio de Zigofiláceas (Larrea y Bulnesia) acompañadas de Leguminosas y Cactáceas (Cabrera, 1994). Los otros tipos de vegetación son de carácter edáfico y se asocian a condiciones alcalinas (estepas halófilas), humedad del suelo (vegetación riparia) y contenido de arena (estepas psamófilas) (Morello, 1958).

Desde un punto de vista fitogeográfico el Monte comprende tres distritos: Distrito Norte extendido desde Salta a La Pampa, Distrito Central restringido al valle Uspallata-Calingasta (entre Mendoza y San Juan) y Distrito Sur extendido desde el sur del Río Colorado (Rio Negro) hasta la Península de Valdez y Punta Ninfas (Chubut) (Roig et al., 2009).

En la Provincia de La Rioja (Argentina), la región del Monte, se extiende por el sector centrooeste (Dptos. Arauco, Castro Barros, Chilecito, Famatina, Felipe Varela, General de Lamadrid, Independencia, Sanagasta, San Blas de los Sauces y Vinchina), ocupando valles intermontanos, conos de deyección, fondos de quebradas y serranías bajas. El conocimiento sobre la flora del Monte en la Rioja es muy escaso y sólo se dispone de descripciones florísticas puntuales (Hosseus, 1916, 1921; Hunziker, 1952; Abraham de Vázquez, 1979; Aceñolaza, 1996).

Los ecosistemas naturales de la Rioja, como en todo el planeta, han experimentado cambios notables en la cobertura y uso del suelo, durante los últimos 50 años (MEA, 2005b). Uno de los factores de cambios más importantes ha sido la expansión de la agricultura (Cabido, 2008; Phalan et al., 2013). Esta actividad es responsable de la pérdidad de hábitat y fragmentación de los ecosistemas naturales (Múgica de la Guerra et al., 2002; Salvador, 2010; García, 2011; Bizama et al., 2011), así como de la degradación de los suelos (Pérez Vázques \& Landero Sánchez, 2009) y constituye la principal amenaza para la diversidad biológica en la actualidad (MEA, 2005b; Cabido, 2008; Phalan et al., 2013).

El cultivo del olivo (Olea europaea) es una de las principales actividades económicas de La Rioja (Cáceres et al., 2009), y su superficie cultivada representa cerca del $70 \%$ de la superficie agrícola total (Searles et al., 2011). La expansión del olivo produjo el desmonte de extensas superficies con vegetación arbustiva (Searles et al., 2011). Actualmente este proceso es notable en el valle Antinaco-Los Colorados (Fig. 1A, B), segundo centro de producción de olivos de la provincia (Cáceres et al., 2009).

Si bien no hay estudios específicos sobre el ritmo de expansión de la agricultura extensiva en el valle, el cultivo de olivo ha mostrado un crecimiento continuo en Argentina (Vita Serman \& Matías, 2013) y su producción se prevé que duplicará hacia el año 2020 (INTA, 2012).

La situación planteada hace necesaria la adopción de medidas inmediatas para regular las actividades productivas y proteger los ecosistemas naturales del valle. Un punto de partida es generar información sobre la biodiversidad del valle que pueda servir de base para priorizar y resguardar áreas de este ecosistema, así como para establecer un plan de conservación y monitoreo a largo plazo. Al presente, 

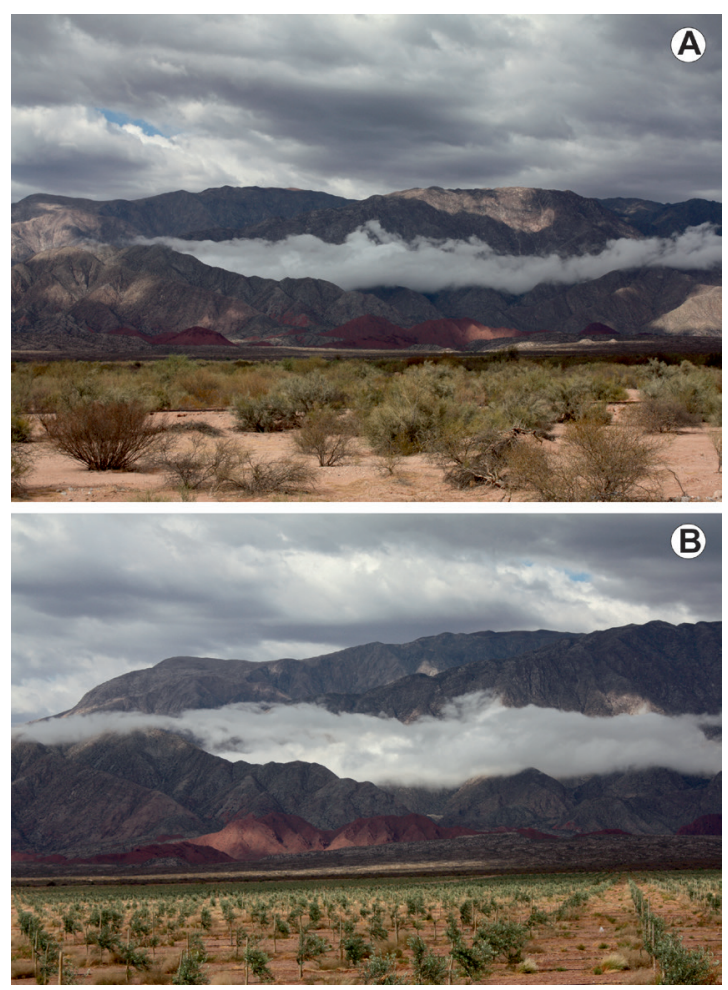

Fig. 1. Cambios en el paisaje producidos por la expansión del cultivo de olivo en Catinzaco (Dpto. Chilecito), durante el período 2007-2010. A: Estepa arbustiva. B: Plantaciones de olivo jóvenes. Fotos: O. Varela.

no hay estudios específicos sobre la vegetación del valle. La contribución botánica más relacionada por su proximidad geográfica, corresponde a una reseña fitogeográfica sobre la Sierra de Famatina (Aceñolaza, 1996).

En este sentido, el presente estudio proporciona un inventario exhaustivo de la diversidad de plantas vasculares y formas de vida del valle AntinacoLos Colorados, y describe la composición de especies asociadas a los distintos tipos de vegetación representados.

Se analiza la composición específica de plantas vasculares nativas e introducidas que crecen espontáneamente, identificando los endemismos de nivel local, regional y nacional. El presente trabajo cubre un vacío de información importante en la región, y aporta datos potencialmente útiles para planificar acciones vinculadas con la recuperación, conservación y manejo sustentable de la flora local.

\section{Materiales y Métodos}

Área de estudio

El valle Antinaco-Los Colorados $\left(\approx 3.000 \mathrm{~km}^{2}\right)$ se localiza en el sector centro oeste de la Provincia de La Rioja, noroeste de Argentina, entre los $28^{\circ} 50^{\prime}-29^{\circ} 57^{\prime} \mathrm{S}$ y $\operatorname{los} 67^{\circ} 23^{\prime}-67^{\circ} 06^{\prime} \mathrm{W}$ (Fig. 2). Abarca una extensión aproximada de $128 \mathrm{~km}$ de largo x $28 \mathrm{~km}$ de ancho (en su parte más extensa), y se extiende a través de los departamentos Famatina, Chilecito e Independencia, en su extremo norte. Está circunscrito entre las cadenas montañosas del Velasco (4.100 msm), al este y Famatina (6.100 $\mathrm{msm})$, al oeste, que corren aproximadamente paralelas con dirección predominante norte-sur. El límite norte del valle lo establece la localidad de Antinaco y el límite sur los Mogotes Colorados.

Geomorfológicamente el valle es una depresión intermontana de origen tectónico y drenaje endorreico, recubierta por aluviones y con grandes depósitos de agua subterránea (Rosa \& Mamaní, 2000). El paisaje posee un relieve plano con inclinación gradual y continua norte-sur, con una altitud máxima de $1100 \mathrm{msm}$ en el sector norte, próximo a Antinaco y una mínima de $660 \mathrm{msm}$ en el sector sur (Los Colorados). Los suelos corresponden al Orden de Los Entisoles. Son, en general, de textura franco arenosa, arenosa (con abundantes clastos en áreas de conos aluviales), muy permeables, de profundidad variable, con escaso desarrollo del perfil, sin estructura, pobres en materia orgánica y nutrientes (PASMA-Pcia. La Rioja, 2001).

De acuerdo a la clasificación de Köppen el clima del valle es de tipo (B.S.h.w) desértico, seco, cálido, con notable amplitud térmica anual. Las lluvias son escasas, el 75\% concentradas en verano (diciembremarzo) y una larga estación seca en invierno (Sosa, 2000). Los promedios anuales de precipitación oscilan entre 100 y $200 \mathrm{~mm}$. Los máximos y mínimos promedios de temperaturas son $25^{\circ} \mathrm{C}$ y $10^{\circ} \mathrm{C}$, respectivamente (PASMA - Pcia. La Rioja, 2001). Los principales ríos que surcan el valle (Capayán, Los Sarmientos, Vichigasta), provienen de la Sierra de Famatina.

El tipo de vegetación dominante es la estepa de arbustos esparcidos, caracterizado por plantas leñosas bajas, muy ramificadas desde la base y con alturas de entre 1-4, $5 \mathrm{~m}$ (Varela, obs. pers.). La composición de especies es característica de la 


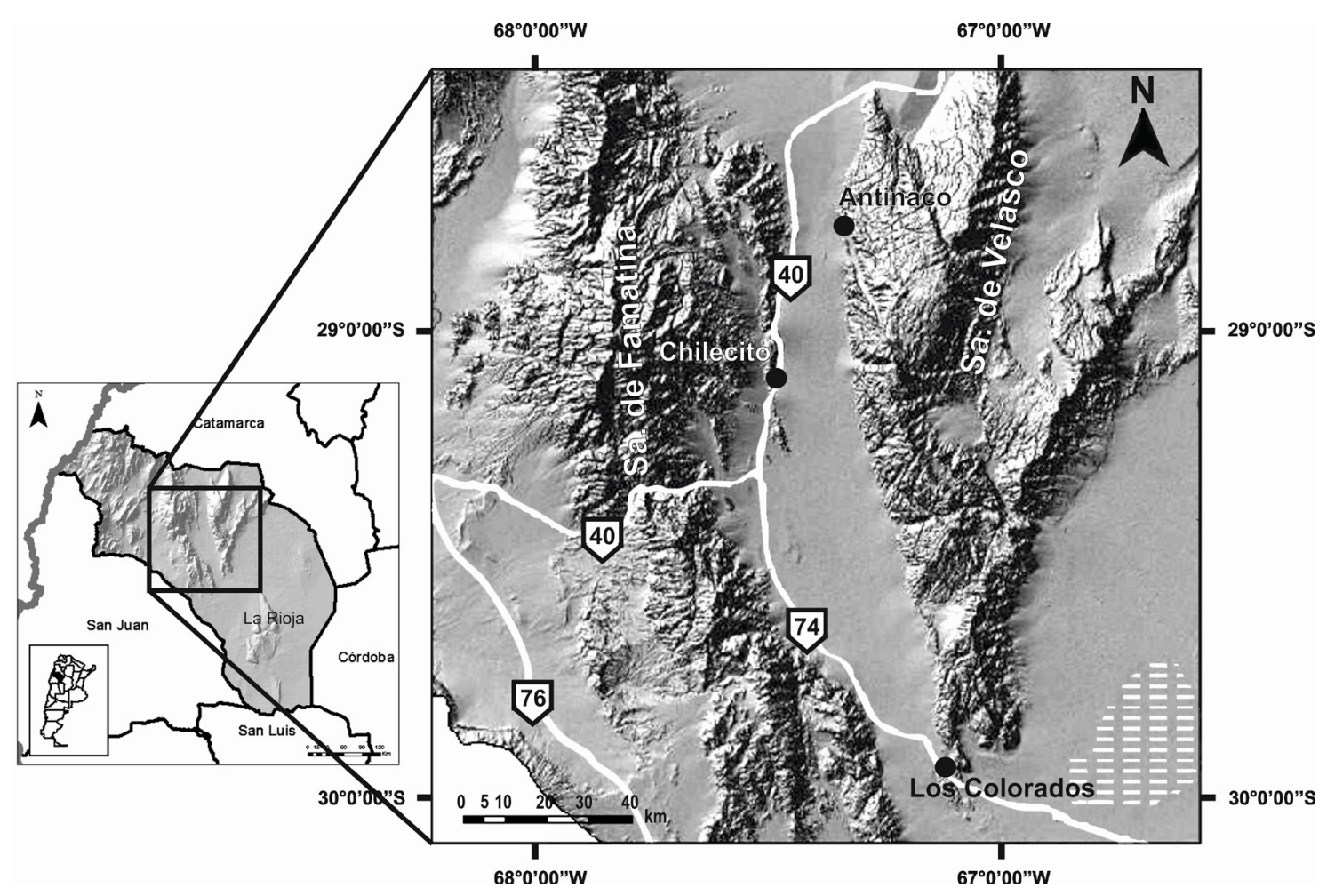

Fig. 2. Ubicación geográfica del Valle Antinaco-Los Colorados, Provincia de La Rioja, Argentina.

Provincia Fitogeográfica del Monte (sensu Cabrera, 1994), con gran uniformidad florística y predominio de jarilla (Larrea cuneifolia) y la retama (Bulnesia retama) (Varela, Obs. pers.). En el extremo sur del valle, donde confluyen las aguas de ríos y arroyos que drenan de las grandes Sierras (Rosa \& Mamaní, 2000), la vegetación es heterogénea debido a la presencia de comunidades de halófitas y especies que ingresan del Chaco árido (Varela \& Jaime, 2010). El valle es atravesado longitudinalmente por las Rutas Nacionales 40 y 74 que se unen en la localidad de Nonogasta. Las localidades con mayor población del Valle son Chilecito, Nonogasta, Vichigasta y Antinaco.

\section{Colecta de material, herborización y determinación}

Durante el período 2006-2015 se realizaron 90 viajes de recolección de plantas vasculares, abarcando todas las estaciones del año, particularmente las estaciones de reproducción y crecimiento (primavera-verano). Se recolectaron todas las especies que crecen espontáneamente en el valle; exceptuando las especies deliberadamente cultivadas. La recolección de especies se realizó principalmente a lo largo del eje longitudinal del valle (norte-sur), a través de las Rutas Nacionales $\mathrm{N}^{\circ} 40$ y 74, en un tramo de $140 \mathrm{~km}$, y también en las Rutas provinciales Los Peregrinos y La Producción. Se seleccionaron áreas de vegetación natural con escasa alteración humana en los Departamentos Chilecito y Famatina, donde se establecieron 11 sitios de recolección, cercanos a las localidades de Antinaco, Capayán, San Nicolás, Los Sarmientos, Malligasta, Chilecito, Nonogasta, Vichigasta, Pulo, Catinzaco y Los Colorados. En cada sitio se recorrió aproximadamente $1 \mathrm{~km}^{2}$ relevando los tipos de vegetación representados. Las áreas que conectan los sitios, particularmente los márgenes de las rutas, fueron también objeto de colecta.

Las zonas modificadas o con vegetación ruderal (márgenes de rutas, vías férreas y canales de riego) fueron objeto de una exhaustiva inspección debido a que son las vías principales de introducción de especies vegetales adventicias (Trombulak \& Frissell, 2000). En cada tipo de vegetación se recolectaron de 3 a 5 ejemplares reproductivos de 


\section{O. Varela et al. - Diversidad de plantas vasculares de Antinaco-Los Colorados}

todas las especies de plantas vasculares (excepto cactáceas) y se anotaron las especies presentes en los distintos tipos de vegetación.

El material fue herborizado, secado en estufa $\mathrm{y}$ acondicionado, siguiendo procedimientos convencionales. La determinación taxonómica se realizó empleando literatura pertinente (floras regionales, flora de Argentina) y corroborando con ejemplares de referencia depositados en el Herbario LIL, determinado por especialistas. Los ejemplares fueron incorporados al herbario de la Fundación Miguel Lillo. La nomenclatura de las plantas silvestres, así como su origen y endemismos fue asignada consultando el Catálogo de las Plantas Vasculares del Cono Sur (Zuloaga et al., 2008) en su versión actualizada online. Las siglas de los autores de los nombres científicos fueron consultadas de Brummitt \& Powell (1992).

Con la información recopilada, se elaboró una lista de las especies encontradas, ordenada por familia botánica, donde se consignan la forma de vida, el origen (cosmopolita, nativa o introducida), los endemismos de nivel local, regional y nacional, los tipos de ambientes donde se encuentran, las nuevas citas para la provincia de La Rioja y el número de ejemplar de herbario de referencia. El término introducido designa a las especies no nativas del país que han ingresado (por semillas u órganos vegetativos) de modo accidental o voluntario por el hombre. La categorización de endemismos se realizó siguiendo los criterios de Cantero et al. (2011), adecuados al sistema de estudio. Se considera especie endémica local a la restringida a la provincia de La Rioja, endémica regional a la distribuida en las provincias de La Rioja, Córdoba, Cuyo y NOA y endémica nacional a la de amplia distribución en Argentina. Se ilustran mediante fotografías los cuatro tipos de vegetación relevados.

\section{Formas de vida}

Cada especie fue asignada a una forma de vida siguiendo la clasificación de Raunkiaer (1934), la cual se basa en la posición y grado de protección de las yemas de renuevo durante el período desfavorable (frío, aridez). Las formas de vidas fueron definidas según criterios próximos a los concebidos originalmente por Raunkiaer (1934), con modificaciones propuestas por Galán de Mera et al. (1999): 1) Fanerófitos: plantas con yemas vegetativas por encima de los $50 \mathrm{~cm}$ de la superficie del suelo), 2) Caméfitos: plantas con yemas vegetativas entre 15 y $50 \mathrm{~cm}$ sobre la superficie del suelo, 3) Hemicriptófitos: plantas herbáceas perennes con yemas vegetativas a menos de $15 \mathrm{~cm}$ del suelo 4) Geófitos: plantas herbáceas perennes con yemas vegetativas por debajo de la superficie del suelo, 5) Terófitos: Plantas anuales representadas por semillas en la estación desfavorable, 6) Epifitos: plantas herbáceas que usan como soporte a otras plantas, 7) Parásitos: Plantas que subsisten total o parcialmente de otras plantas, 8) Hidrófitos: plantas perennes con raíces bajo el agua.

\section{Resultados}

Diversidad de taxones, origen y endemismos

Se registraron 264 especies correspondientes a 57 familias y 181 géneros, de las cuales 229 son nativas $(86,4 \%), 33$ son introducidas $(13,2 \%)$ y una es cosmopolita $(0,4 \%)$ (Tabla 1 , Apéndice 1$)$. Del total de taxones, 263 corresponden a Angiospermae (213 Dicotyledoneae y 49 Monocotyledoneae), uno a Gymnospermae y uno a Helechos (Tabla 1). Las 10 familias más diversas aportaron el $65 \%$ de las especies y fueron, en orden decreciente de importancia, Asteraceae (35 spp., 13\%), Poaceae (32 spp., 12\%), Fabaceae (19 spp., 7\%), Solanaceae (19 spp., 7\%), Cactaceae (16 spp., 6\%), Chenopodiaceae (14 spp., 5\%), Bromeliaceae (12 spp., 5\%), Verbenaceae (10 spp., 4\%), Malvaceae (9 spp., 3\%) y Euphorbiaceae (8 spp., 3\%). Por otra parte, el $30 \%$ de las familias incluyeron de 2

Tabla 1. Diversidad taxonómica de grandes grupos de plantas vasculares en el Valle Antinaco-Los Colorados (La Rioja, Argentina).

\begin{tabular}{|lccc|}
\hline \multicolumn{1}{|c}{ Grupos } & Familias & Géneros & Especies \\
Helechos & 1 & 1 & 1 \\
Spermatophyta & & & \\
Gymnospermae & 1 & 1 & 1 \\
Angiospermae & & & \\
$\quad$ Dicotyledoneae & 50 & 149 & 213 \\
Monocotyledoneae & 5 & 30 & 49 \\
Total & $\mathbf{5 7}$ & $\mathbf{1 8 1}$ & $\mathbf{2 6 4}$ \\
\hline
\end{tabular}


a 7 especies mientras que el 53\% restante una sola especie. Asimismo, el $39 \%$ de las familias estuvieron representadas por 2-30 géneros mientras que el $61 \%$ restante, por un solo género. Cabe resaltar que solo 3 familias (Asteraceae, Poaceae, Fabaceae) incluyeron más de 10 géneros, y aportaron el 35\% de los géneros (64) encontrados en el valle. Los géneros mejor representados fueron Tillandsia (10 spp.) y Solanum (6 spp.), seguidos por Atriplex, Lycium y Prosopis, cada uno con 5 especies. La mayoría de los géneros $(75 \%)$ presentaron una sola especie. Las plantas introducidas, corresponden a 14 familias, principalmente Poaceae (8 spp.), Asteraceae (5 spp.), Brassicaceae (5 spp.) y Chenopodiaceae (5 spp.).

En el valle están representadas 84 especies endémicas de Argentina, el $49 \%$ de las cuales pertenecen a las familias Cactaceae (13 spp.), Asteraceae (9 spp.), Solanaceae (7 spp.), Chenopodiaceae (6 spp.) y Euphorbiaceae (6 spp.). Considerando la escala de endemismos, una sola especie $(1 \%)$ está restringida a la provincia de La Rioja (Gymnocalycium aff. mazanense), 31 especies $(37 \%)$ son endémicas regionales y 52 $(62 \%)$ son endémicas nacionales. La mayoría de las plantas endémicas son hierbas (45 spp., 54\%,) o arbustos (37 spp., 44\%).

Caracterización florística de los tipos de vegetación representados en el valle

El valle incluye cuatro tipos principales de vegetación (Fig. 3), según su fisonomía, características edáficas y composición de especies:

Estepa arbustiva típica: Es la unidad de vegetación de mayor extensión, desarrollada sobre suelos arenosos y conos aluviales, de gran homogeneidad florística. Fisonómicamente está definida por arbustos y árboles xerófilos (micrófilos, áfilos, inermes o espinescentes, resinosos), de porte mediano-alto (1,5-4 $\mathrm{m})$ dispersos, que dejan grandes espacios de suelo al descubierto. Las especies más representativas de mayor distribución y abundancia a través de valle son: Bulnesia retama, Larrea cuneifolia (Zigophyllaceae), Cercidium praecox, Prosopis torquata, Senna aphylla, Zuccagnia punctata (Fabaceae), Opuntia sulphurea, Tephrocactus articulatus, Echinopsis leucantha, Gymnocalycium spp. (Cactaceae) y Tricomaria usillo (Malphigiaceae). En las zonas de
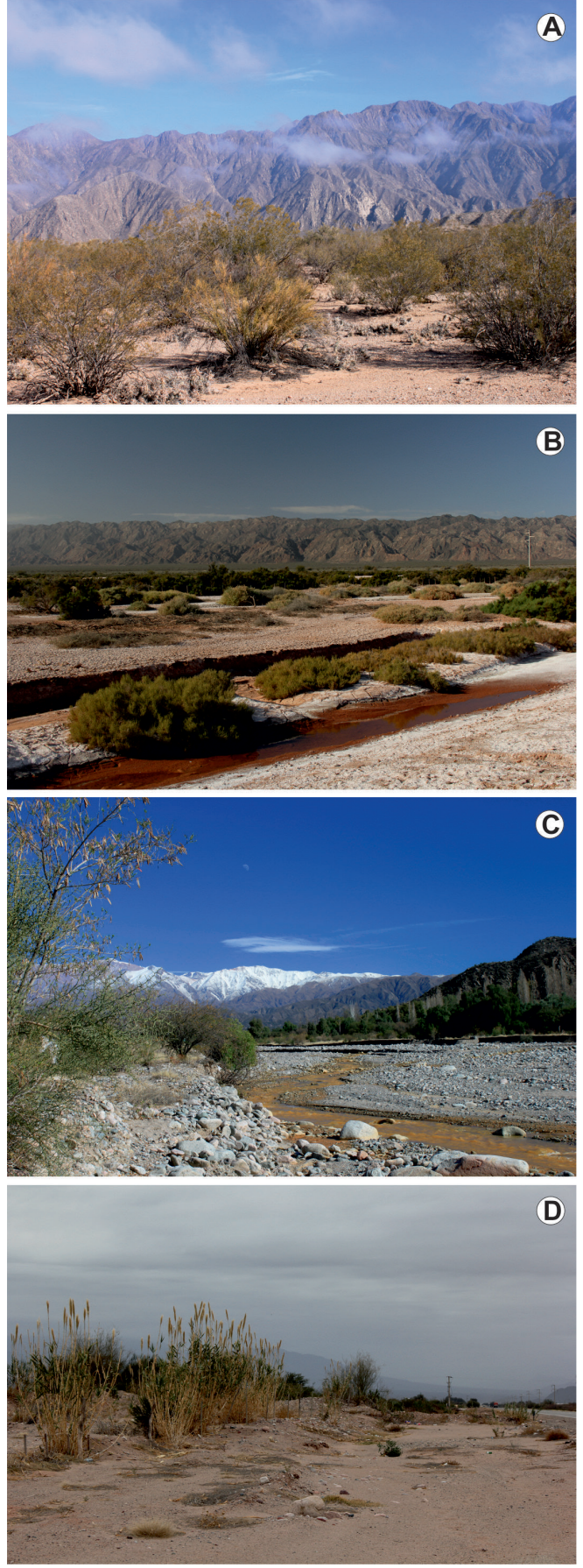

Fig. 3. Tipos principales de vegetación del valle Antinaco-Los Colorados. A: Estepa arbustiva típica, próxima a Antinaco. B: Estepa arbustiva halófila, cercana a Los Colorados. C: Vegetación riparia (Río Los Sarmientos). D: Vegetación ruderal (márgenes de la Ruta Nacional No 74, próximo a Vichigasta). Fotos: O. Varela. 


\section{O. Varela et al. - Diversidad de plantas vasculares de Antinaco-Los Colorados}

abanicos aluviales, surcada por numerosos arroyos temporarios, la leñosa de mayor abundancia en los márgenes de cauces es Acacia gilliesii (Fabaceae).

Entre las hierbas más abundantes se destacan Aristida adscensionis, A. mendocina, Bouteloua aristidoides, Chloris virgata, Cottea pappophoroides, Digitaria californica, Pappophorumphillippianum, Setariamacrostachya, Sporobolus pyramidatus, Trichloris crinita (Poaceae), Allionia incarnata (Nyctaginaceae), Heliotropium chrysanthum (Boraginaceae), Amaranthus standleyanus, Gomphrena tomentosa (Amaranthaceae), Bidens subalternans, Flaveria bidentis (Asteraceae), Portulaca confertifolia y $P$. grandiflora (Portulacaceae), Euphorbia catamarcensis, E. ruiz-leali (Euphorbiaceae), Gaya parviflora (Malvaceae). Entre las enredaderas la más frecuente es Cucurbitella asperata (Cucurbitaceae), y entre las epífitas la especie más abundante es Tillandsia duratii.

Estepa arbustiva halofila: Lo conforman arbustos compactos $(<2 \mathrm{~m})$, dispersos, inermes, adaptados a suelos salobres, de textura fina, mal drenados, donde predominan pocas especies de la familia Chenopodiaceae. Los taxones dominantes son Suaeda divaricata, Allenrolfea vaginata, Atriplex lampa, A. spegazzinii, A. argentina, A. cordobensis, (Chenopodiaceae), Maytenus vitis-idaea (Celastraceae), Geoffroea decorticans, Prosopis strombulifera, Prosopis sericantha (Fabaceae) y Cyclolepis genistoides (Asteraceae). Entre las hierbas predominan Ehretia cortesia. Heliotropium chrysanthum, H. curassavicum (Boraginaceae), Sporobolus phleoides S. pyramidatus, S. rigens, Distichlis spicata (Poaceae), Sesuvium portulacastrum, Trianthema argentina (Aizoaceae), Solanum euacanthum, Sclerophylax kurtzii (Solanaceae), Gomphrena tomentosa (Amaranthaceae), Euphorbia ruiz-lealii (Euphorbiaceae), Portulaca echinosperma (Portulacaceae) y entre las volubles, Grahamia bracteata (Anacampserotaceae). Son características de este ambiente Pterocactus tuberosus y Tephrocactus halophilus (Cactaceae).

Vegetación riparia: Comprende la vegetación asociada a los cursos de aguas temporales (ej. río Los Sarmientos, río Capayán y arroyos), tanto de terrazas marginales no inundables, como del cauce de inundación. En las márgenes no inundables se desarrolla un arbustal denso, compuesto por Acacia caven, Bredemeyera colletioides, Tecoma garrocha, Tessaria dodoneifolia, Acacia aroma, Celtis ehrenbergiana, Prosopis chilensis y Acacia visco. Caracterizan a los cauces temporalmente secos Bredemeyera colletioides, Porophyllum obscurum, Parthenium hysterosphorus, Austrobrickellia patens, Hyalis argentea, Senecio pinnatus, Tessaria dodoneifolia, Zexmenia buphtalmiflora (Asteraceae), Nicotiana glauca, Nicotiana noctiflora, Petunia axillaris, Solanum annuum (Solanaceae), Argemone subfusiformis (Papaveraceae), Mentzelia albescens, M. parvifolia (Loasaceae), Tecoma garrocha (Bignoniaceae), Glandularia mendocina (Verbenaceae), Gaya parviflora, Herissantia crispa y Sida argentina (Malvaceae).

Vegetación ruderal: Comprende las plantas que habitan en terrenos modificados por la actividad humana (márgenes de caminos, vías férreas, canales de riego) mayormente representadas por especies herbáceas. Los bordes de rutas o zonas adyacentes a las rutas son áreas de continua alteración antrópica que actúan como fuente de propágulos de plantas adventicias.

Las banquinas y terrenos próximos a las Rutas Nacionales $\mathrm{N}^{\circ} 40$ y 74 (que atraviesan el valle por su parte media), así como las Rutas de La Producción y de Los Peregrinos son principales vías de introducción y dispersión de plantas en el valle. Caracterizan a este tipo de vegetación: Arundo donax, Cenchrus ciliaris, C. myosuroides, Cynodon dactylon, Digitaria ciliaris, Paspalum urvillei, Sorghum halepense, Sporobolus maximus (Poaceae), Eruca vesicaria, Hirschfeldia incana, Lepidum bonariensis, Sisymbrium irio, Rapistrum rugosum (Brassicaceae), Atriplex suberecta, Bassia scoparia, Chenopodium album, C. murale, Dysphania ambrosioides, Salsola kali (Chenopodiaceae), Alternanthera pungens, Amaranthus muricatus, A. viridis, Gomphrena martiana (Amaranthaceae), Baccharis glutinosa, B. salicifolia, Centaurea melitensis, Cirsium vulgare, Conyza bonariensis, Flaveria bidentis, Sonchus oleraceus, Taraxacum officinale, Tessaria absinthioides, Zinnia elegans (Asteraceae) y Portulaca oleracea (Portulacaceae). 
La contribución de cada ambiente a la riqueza del valle se muestra en la Tabla 2. La estepa arbustiva típica y la vegetación ruderal son los tipos de vegetación más diversos, e incluyen a la mayoría de las especies representadas en el valle.

El único endemismo provincial (Gymnocalycium aff. mazanense), fue encontrado asociado a suelos pedregosos de conos aluviales, en los sitios de Capayán y Los Sarmientos.

\section{Formas de vida}

La flora vascular del valle está representada por ocho formas de vida correspondientes a la clasificación de Raunkiaer (1934) (Fig. 4A). Los Fanerófitos, representados principalmente por arbustos, son las formas de vida más representativas de la región y las que determinan la fisonomía del paisaje. Representan el 28 $\%$ de las especies y caracterizan a las estepas de jarilla y retama y halófila del valle. De similar importancia en diversidad son los Terófitos (hierbas anuales) y en tercer lugar los Hemicriptófitos (hierbas perennes). En la estepa típica (Fig. 4B) los Terófitos ocupan el tercer lugar en importancia, precedido por los Fanerófitos y Hemicriptófitos.

La frecuencia de formas de vida para las familias más diversas se muestra en la Figura 5. Las familias Asteraceae y Solanaceae registran la mayor diversidad de formas de vida, dominadas por Terófitos y Fanerófitos.

En la familia Poaceae, integrada casi totalmente por plantas herbáceas, los Hemicriptófitos fueron los mejor representados, seguidos de los Terófitos y Geófitos. En otras familias diversas y numéricamente abundantes como Fabaceae y Cactaceae, las formas dominantes fueron los Fanerófitos (Fabaceae) y Caméfitos (Cactaceae). Los taxones de Chenopodiaceae, característicos y diversos en ambientes halófilos, están representados principalmente por Fanerófitos y Terófitos.

\section{Citas nuevas para la Provincia de La Rioja}

La exploración exhaustiva de los distintos tipos de vegetación permitió encontrar especies no citadas para la provincia de La Rioja. Se registran para la flora de la provincia dieciocho nuevas citas en el Departamento Chilecito, que
Tabla 2. Número de especies de plantas vasculares según el tipo de vegetación. Ref: C. Cosmopolita, I. Introducida, E. Endémica, N. Nativa.

\begin{tabular}{|lcccccc|}
\hline Tipos de vegetación & C & I & E & N & Total \\
\hline Estepa típica & 1 & 5 & 56 & 78 & 140 \\
Estepa halófita & & 1 & 23 & 15 & 39 \\
Vegetación riparia & & 10 & 32 & 76 & 118 \\
Vegetación ruderal & 1 & 32 & 24 & 91 & 148 \\
\hline
\end{tabular}
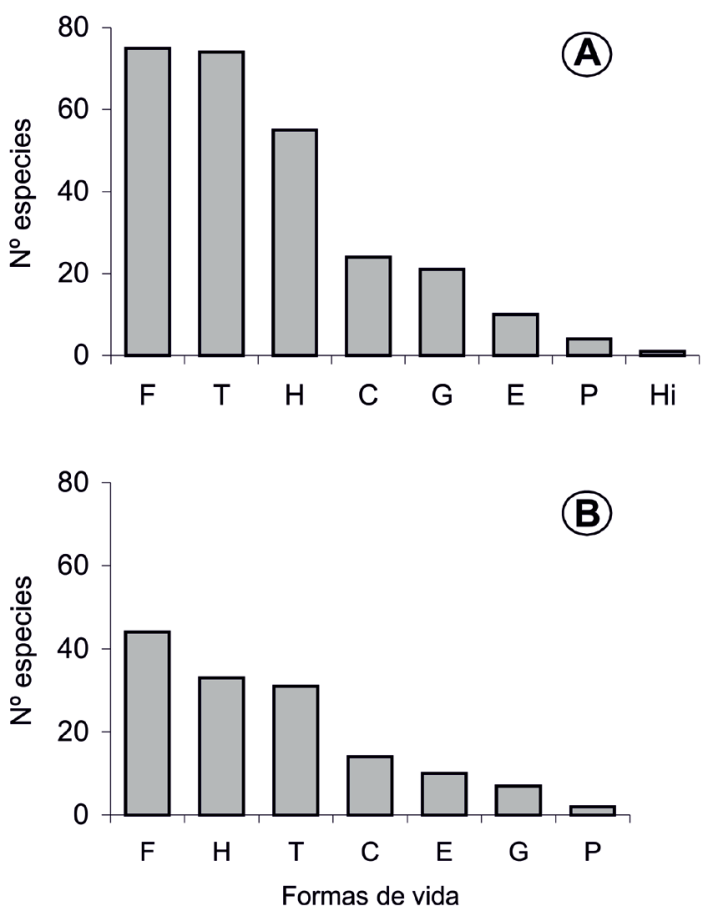

Fig. 4. Distribución del número de especies por formas de vida para el valle Valle Antinaco-Los Colorados (A) y para la Estepa arbustiva típica (B). Referencias: C. Caméfitos, E. Epífitos, F. Fanerófitos, G. Geófitos, H. Hemicriptófitos, Hi. Hidrófitos, P. Parásitos, T. Terófitos.

se listan a continuación. El material de referencia para cada una de ellas, se encuentra depositado en el Herbario de la Fundación Miguel Lillo (LIL), en la provincia de Tucumán. 


\section{Anagallis arvensis L., Sp. Pl. 1: 148. 1753.}

Iconografía. Kiesling, en Cabrera (ed.), Fl. Prov. Jujuy 8: 21, f. 9 A-M, 1983.

Distribución geográfica y hábitat: Originaria de Europa, se ha asilvestrado en las zonas templadas del país. Es una especie invasora en terrenos modificados (Kiesling, 1983; Juárez, 1996). En el área de estudio se la encuentra en banquinas, márgenes de canales de riego y acequias.

Material estudiado. Los Sarmientos, acequia frente a Campus de la UNdeC, 06-VIII-2014, O.Varela 208 (LIL).

2. Bassia scoparia (L.) A.J. Scott, Feddes Repert. 81(2-3): 108. 1978. Basónimo: Chenopodium scoparium $\mathrm{L}$.

Iconografia. Soriano, Revista Argent. Agron. 12: 52-53, 1945.

Distribución geográfica y hábitat. Originaria de Eurasia. Pionera en suelos erosionados, maleza de cultivos (Giusti, 1967; Mulgura, 1994).

Material estudiado. Vichigasta, Márgenes RN No 74, 07-VIII-2014, O. Varela 99 (LIL).

3. Bidens pilosa L., Sp. Pl. 2: 832. 1753.

Iconografia. Cabrera, en Cabrera (ed.), Fl. Prov. Jujuy 13(10):416, f. 174 F-N, 1978.

Distribución geográfica y hábitat. Especie pantropical, muy difundida en terrenos modificados o alterados (Cabrera, 1978). En el área de estudio habita en bordes de rutas, canales de riego, acequias y campos de cultivos (Novara \& Gutierrez, 2010).

Material estudiado. Los Sarmientos, acequia frente a campus de la UNdeC, 31-III-2012, O. Varela 27 (LIL).

\section{Boerhavia diffusa L., Sp. Pl. 1: 3. 1753.}

Iconografia. Múlgura, en Troncoso \& Bacigalupo (ed), Fl. Il. Entre Ríos 6(3): 208, f. 95 A-D, 1987.

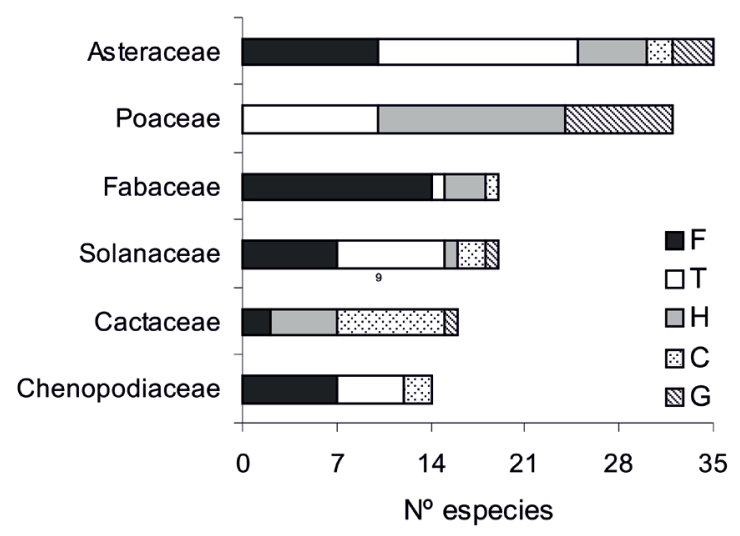

Fig. 5. Frecuencia de formas de vida para las seis familias de plantas vasculares más diversas del Valle Antinaco-Los Colorados (Pcia. La Rioja, Argentina). Referencias: C. Caméfitos, F. Fanerofitos, G. Geófitos, H. Hemicriptófitos, T. Terófitos.

Distribución geográfica y hábitat. Distribuida en regiones tropicales y subtropicales de ambos hemisferios (Múlgura, 1987). En Argentina se extiende por las provincias del norte y centrales hasta La Pampa. Habita en terrenos modificados (Lucena, 1993).

Material estudiado. La Puntilla, zona de bifurcación de caminos La Puntilla - Samai Huasi, 05-IV-2013, O. Varela 161 (LIL).

5. Centaurium pulchellum (Sw.) Druce, Fl. Berkshire 342. 1897. Basónimo: Gentiana pulchella Sw.

Iconografia. Fabris, Bol. Soc. Argent. Bot. 4(4): 237, f. 1 N-O, 1953.

Distribución geográfica y hábitat. Es una especie originaria del hemisferio norte, muy difundida en Argentina (Fabris, 1983; Juárez, 1997). En el área de estudio habita en los márgenes de canales de riego y en terrenos cultivados.

Material estudiado. Los Sarmientos, márgenes de canal de riego frente a Campus de la UNdeC, 30-I-2013, O. Varela 139 (LIL). 
6. Cyclospermum leptophyllum (Pers.) Sprague, J. Bot. 61(725): 131. 1923. Basónimo: Pimpinella leptophylla Pers.

Iconografía. Lucena et. al., en Novara (ed.), Fl. Valle de Lerma 6(11): 46, Lám. 16 A-F, 2001.

Distribución geográfica y hábitat. Maleza de regiones templadas en todo el mundo. Es frecuente en suelos arenosos, soleados y húmedos (Lucena et al., 2001).

Material estudiado. Los Sarmientos, márgenes de acequia frente a Campus de la UNdeC, 06-082014, O. Varela 18 (LIL).

7. Diplotaxis tenuifolia (L.) DC., Syst. Nat. 2: 632 633. 1821. Basónimo: Sisymbrium tenuifolium L.

Iconografia. Boelcke \& Marttínez Laborde, en Kiesling (ed.), Fl. San Juan 1: 239, f. 268 A-C, 1994.

Distribución geográfica y hábitat. Originaria de Europa. Es una maleza ampliamente distribuida en áreas con riego (Boelcke \& Martínez-Laborde, 1994).

Material estudiado. Los Sarmientos, Río Los Sarmientos detras de Campus de la UNdeC, 16-III2013, O. Varela 64 (LIL).

8. Hirschfeldia incana (L.) Lagr.-Fossat, Fl. Tarn Garonne 19. 1847. Basónimo: Sinapis incana L.

Iconografia. Boelcke \& Martínez Laborde, en Kiesling (ed.), Fl. San Juan 1: 241, f. 271 A-B, 1994.

Distribución geográfica y hábitat. Originaria de Europa, muy difundida en Argentina como maleza en cultivos invernales y pasturas perennes, como alfalfa (Boelcke \& Martínez-Laborde, 1994).

Material estudiado. Los Sarmientos, Río Los Sarmientos, detrás del Campus de la UNdeC, 16III-2013, O. Varela 66 (LIL).

9. Maytenus vitis-idaea Griseb., Abh. Königl. Ges. Wiss. Göttingen 19: 110. 1874.
Iconografía. Lourteig \& O’Donell, Natura (Buenos Aires) 1(2): 197, 1955.

Distribución geográfica y hábitat. Epecie endémica de Argentina, Bolivia y Paraguay. Habita en suelos salinos de la región chaqueña de Bolivia, Paraguay, norte y centro argentino hasta la región del Monte y en el norte de la Patagonia (Novara, 1993).

Material estudiado. Salinas entre Catinzaco y Los Colorados, 03-VIII-2014, O. Varela 90 (LIL).

10. Medicago polymorpha L., Sp. Pl. 2: 779. 1753. Iconografía. Burkart, en Troncoso \& Bacigalupo (ed.), Fl. Il. Entre Ríos 6 (3a): 648, f. 301 A-B, 1987.

Distribución geográfica y hábitat. Originaria del Mediterráneo (Burkart, 1987).

Material estudiado. Los Sarmientos, acequia frente a Campus de la UNdeC, 14-V-2014, O. Varela 129 (LIL).

11. Plantago lanceolata L., Sp. Pl. 1: 113-114. 1753.

Iconografia. Rahn, en Burkart (ed.), Fl. Il. Entre Ríos, 6(5): 586, f. 298 A-H, 1979.

Distribución geográfica y hábitat. Originaria de Eurasia y muy difundida en todo el mundo. Habita en suelos húmedos, fértiles, arenosos y algo sombreados, comúnmente en borde de caminos, cunetas de vías férreas, baldíos, jardines y en proximidades a viviendas rurales (Rahn, 1979; Tolaba \& Fabbroni, 1998).

Material estudiado. Los Sarmientos, márgenes de canal de Riego frente a campus de la UNdeC, 14-V-2014, O. Varela 169 (LIL).

12. Prosopanche bonacinae Speg., Comun. Mus. Nac. B. Aires 1(1): 19-22. 1898.

Iconografia. Kiesling, en Kiesling (ed.), Fl. San Juan 1: 70, f. 55 A-F, 1994.

Distribución geográfica y hábitat. Presenta una 


\section{O. Varela et al. - Diversidad de plantas vasculares de Antinaco-Los Colorados}

amplia distribución geográfica, desde la Patagonia hasta el Paraguay y sur de Brasil. Es parasita de raíces de distintas familias de plantas nativas (Anacardiaceae, Chenopodiaceae, Rhamnaceae, Malvaceae, Apiaceae, Solanaceae, Asteraceae, Fabaceae) y cultivadas (Dawson, 1967; Kiesling, 1994). En el área de estudio se la encontró parasitando raíces de Fraxinus.

Material estudiado. La Puntilla, sobre raíces de Fraxinus spp, 21-III-2013, O. Varela 142 (LIL).

13. Rapistrum rugosum (L.) All., Fl. Pedem. 1: 257. 1785. Basónimo: Myagrum rugosum L.

Iconografia. Boelcke \& Marttínez Laborde, en Kiesling (ed.), Fl. San Juan 1: 211, f. 228 A-B, 1994.

Distribución geográfica y hábitat. Especie europea, considerada maleza, ampliamente difundida en la región agrícola bajo riego y en áreas de secano (Boelcke \& Martínez-Laborde, 1994).

Material estudiado. Los Sarmientos, Río Los Sarmientos, detrás de Campus de la UNdeC, 08VIII-2014, O. Varela 68 (LIL).

\section{Salsola kali L., Sp. Pl. 1: 222. 1753.}

Iconografia. Tolaba, en Novara (ed.), Fl. Valle de Lerma 7(18): 11, Lám. 3 A-E, 2006.

Distribución geográfica y hábitat. Especie de origen euroasiático, cosmopolita. Es invasora de cultivos y suelos modificados (Tolaba, 2006).

Material estudiado. Los Sarmientos, márgenes de acequia frente a Campus de la UNdeC, 08-VIII2014, O. Varela 105 (LIL).

15. Schinus polygamus (Cav.) Cabrera, Obr. Centen. Mus. La Plata 2: 269. 1937. Basónimo: Amyris polygama Cav.

Iconografia. Cabrera, Revista Mus. La Plata 2(6): 26, f. 7A-C, 1938.

Distribución geográfica y hábitat. Especie endémica de Argentina, Brasil, Chile, Paraguay y
Uruguay (Martínez Carretero, 2009).

Material estudiado. Los Sarmientos, márgenes de canal de riego frente a Campus UNdeC, 14-VIII2008, O. Varela 16 (LIL).

16. Sida calchaquiensis Rodrigo, Revista Mus. La Plata, Sec. Bot. 24: 167. 1944.

Iconografia. Krapovickas, en Kiesling (ed.), Fl. San Juan 2: 118, f. 114, 2003.

Distribución geográfica y hábitat. Especie endémica de Argentina de los valles Calchaquies (Salta, Tucumán) San Juan, Corrientes (Krapovickas, 2003).

Material estudiado. Los Sarmientos, frente a Campus de la UNdeC, 15-V-2015, O. Varela 154 (LIL).

17. Tamarix ramosissima Ledeb., Fl. Altaic.1: 424-426. 1829.

Iconografia. Kiesling, en Kiesling (ed.), Fl. San Juan 2: 138, f. 139 A-E, 2003.

Distribución geográfica y hábitat. Es una especie europea introducida y naturalizada en todo el país. Crece en terrenos húmedos y arenosos, frecuentemente asociadas a los cauces de ríos y en las cunetas de rutas (Kiesling, 2003).

Material estudiado. Vichigasta, márgenes $\mathrm{RN} \mathrm{N}^{\mathrm{o}}$ 74, 11-XI-2006, O. Varela 233 (LIL).

18. Tephrocactus halophilus (Speg.) Backeb., en Backeb. \& Knuth, Kaktus-ABC. 11, 1936 - Opuntia halophila Speg.

Iconografia. Kiesling \& Meglioli, en Kiesling (ed.), Fl. San Juan 2: 172, f. 171 A-B, 2003.

Distribución geográfica y hábitat. Especie endémica de Argentina (San Juan y San Luis). Habita en suelos salinos de la región del Monte entre los 1000 y 1500 msm (Kiesling \& Meglioli, 2003).

Material estudiado. Pulo, áreas salinas frente a $\mathrm{RN} \mathrm{N}^{\circ}$ 74, 23-IV-2015, O. Varela 85 (LIL). 


\section{Discusión}

El presente estudio aporta el primer inventario completo de plantas vasculares realizado para un área extensa del Monte de La Rioja.

Previo a este estudio, el listado florístico más diverso realizado para el Monte de La Rioja registra un total de 132 entidades de plantas vasculares (Hunziker, 1952). El número de especies encontrado (264) duplica el número de especies conocido (Hunziker, 1952), y representa aproximadamente el $17 \%$ de la diversidad total de plantas vasculares $(\approx 1.530)$ registradas para la provincia (Zuloaga et al., 2008 - versión online actualizada). Este porcentaje incrementa en importancia al relacionarlo con la superficie del valle, la cual representa el 3,3\% de la superficie del territorio provincial $\left(89.680 \mathrm{~km}^{2}\right)$.

En cuanto a los taxones introducidos (33 spp.; 13\%), estos se hallan circunsriptos a sitios modificados o ruderales (cunetas de rutas, canales de riego), áreas que concentran y retienen mayor humedad por períodos más largos, lo que podría explicar por qué estos son infrecuentes en las áreas no perturbadas de la estepa típica.

De acuerdo al estudio de Morello (1958) las familias Fabaceae, Poaceae, Asteraceae y Cactaceae representan los grupos de plantas más diversos de la región del Monte. En el presente estudio las familias Asteraceae y Poáceas fueron las más diversas, seguidas en importancia de las Solanaceae, Fabaceae y Çactaceae.

La vegetación del valle tiene un componente relativamente alto de Asteráceas y Poáceas anuales, si bien, cerca del $26 \%$ de las especies son de naturaleza ruderal. El incremento de los Terófitos a nivel del valle es explicado por estas especies; lo cual no se evidencia en la estepa típica, donde son escasas.

De las 264 especies encontradas en el valle, sólo 140 componen la "estepa arbustiva típica", el tipo de vegetación más extendido del valle.

La mayoría de la nuevas citas corresponden a plantas ruderales que habitan en áreas en constante modificación. En cuanto a las especies endémicas de categorización regional y nacional, atendiendo a su distribución geográfica y de acuerdo a la lista roja de las especies amenazadas de la IUCN (versión online disponible en: http://www.iucnredlist.org/), se puede inferir que ninguna tiene problemas de conservación.
El espectro biológico del valle concuerda con las condiciones climáticas imperantes de regiones áridascálidas (Sudhakar Reddy et al., 2011). El predominio de los Fanerófitos es congruente con el espectro biológico de comunidades del Parque Provincial Ischigualasto en San Juan, Argentina (Márquez et al., 2005). La abundancia de Terófitos refleja una estrategia efectiva para superar las condiciones adversas, en ambientes áridos con altas temperaturas y deficiencia de humedad (Van Rooyen et al., 1990). Los Terófitos son plantas anuales que desarrollan su ciclo vital completo durante períodos cortos de lluvias y permanecen en estado vegetativo en forma de semilla, durante la estación desfavorable (Noy-Meir, 1973).

Otro aspecto de este estudio, y quizás el más importante, es que puede ser un valioso aporte y punto de partida para gestionar la creación de un área protegida en el valle. Sus ecosistemas naturales se encuentran en riesgo cierto de desaparecer debido al avance de los cultivos extensivos (olivo, vid), proceso que por su magnitud y celeridad (Searles et al., 2011) constituye la mayor amenaza para la conservación de la biodiversidad.

\section{Conclusiones}

El valle Antinaco-Los Colorados es un área representativa del Monte Septentrional de Argentina. Concentra aproximadamente el 17\% de la diversidad de plantas vasculares conocida para La provincia de La Rioja en una superficie relativamente reducida. Su flora vascular se compone de un $87 \%$ de especies nativas y un $13 \%$ de introducidas. Las familias más diversas son las Asteraceae, Poaceae, Fabaceae, Solanaceae y Cactaceaae. Incluye 84 endemismos principalmente de Cactaceae, Asteraceae, Solanaceae, Chenopodiaceae y Euphorbiaceae. Las formas de vida dominantes son los Fanerófitos, Terófitos y Hemicriptófitos (21\%).

\section{Agradecimientos}

Los autores agradecen la colaboración de la siguientes personas e instituciones: a Gimena Toledo por su asistencia en la organización del material de referencia, a Patricia Asesor y Griselda Podaza por la elaboración del mapa, a Hugo 
Ayarde por la lectura crítica del manuscrito y valiosos aportes, al personal del Herbario LIL por las facilidades ofrecidas para la consulta de material botánico, y a las instituciones Fundación Miguel Lillo y Universidad Nacional de Chilecito por el apoyo logístico y financiero para los viajes de estudio. Los comentarios y sugerencias de dos revisores anónimos han contribuido a mejorar el manuscrito.

\section{Biblografía}

ABRAHAM DE VÁZQUEZ, E.M. 1979. Geomorfología y biota del valle de Sanagasta (Provincia de La Rioja). Deserta 95-155.

ABRAHAM, E., DEL VALLE H.F., ROIG, F., TORRES, L., ARES, J.O., CORONATO, F., \& R. GODAGNONE. 2009. Overview of the geography of the Monte Desert biome (Argentina). J. Arid Environ.73: 144-153.

ACEÑOLAZA, P.G. 1996. Vegetación. In: F.G. ACEÑOLAZA, F.G., H. MILLER \& A. TOSELLI (eds.), Geología del sistema de Famatina (Münchner Geologische Hefte 19 (Reihe A). pp.19-22. München.

BIZAMA, G., TORREJÓN, F., AGUAYO, M., MUÑOZ, M.D., ECHEVERRÍA, C. \& R. URRUTIA. 2011. Pérdida y fragmentación del bosque nativo en la cuenca del río Aysén (Patagonia-Chile) durante el siglo XXI. Rev. geogr. Norte Gd. 49: 125-138.

BOELCKE, O. \& J.B. MART'INEZ-LABORDE. 1994. Cruciferae. In: R. KIESLING. (ed.), Flora de San Juan 1: 205-244. Vázques Mazzini, Buenos Aires.

BRUMMITT, R.K. \& C.E. POWELL. 1992. Authors of Plant Names. Royal Botanic Gardens, Kew: 1-732.

BURKART, A. 1987. Leguminosae. In: N.S. TRONCOSO \& N.M. BACIGALUPO (eds.), Flora ilustrada de. Entre Ríos, Colecc. Ci. Inst. Nac. Tecnol. Agropecu. 6(3a): 42-738.

CABIDO, M. 2008. Impacto de la agricultura sobre la extensión, distribución y biodiversidad de ecosistemas naturales. In: O. SOLBRIG \& J. ADÁMOLI (coord.), Agro y Ambiente: una agenda compartida para el desarrollo sustentable. Capítulo 7. Foro de la Cadena Agroindustrial.

CABRERA, A.L. 1978. Compositae. In: A.L. CABRERA (ed.), Flora de la Provincia de Jujuy, Colecc. Ci. Inst. Nac. Tecnol. Agropecu. 13(10): 1-726.

CABRERA, A.L. 1994. Regiones Fitogeográficas Argentinas. In: W.F. KUGLER (reimp.), Enciclopedia Argentina de Agricultura y Jardinería 2 (1): 1-85. Ed. A.C.M.E., Buenos Aires.

CÁCERES, R., NOVELlO, R. \& M. ROBERT. 2009. Análisis de la cadena del olivo en Argentina. Estudios socioeconómicos de los sistemas Agroalimentarios y Agroindustriales 2:43-60. Ediciones INTA.

CANTERO, J.J., SFRAGULLA, J.A., NÚÑEZ, C., BONALUMI, A., MULKO, J., AMUCHASTEGUI, A., BARBOZA, G., CHIARINI, F. \& L. ARIZA ESPINAR. 2011. Flora de los afloramientos de mármoles y serpentinitas de las Sierras de Córdoba (Argentina). Kurtziana 36: 11-45.

DARKOH, M.B.K. 2003. Regional perspectives on agriculture and biodiversity in the drylands of Africa. J. Arid Environ. 54:261-279.

DAWSON, G. 1967. Hydnoraceae. In: Fl. Buenos Aires, Colecc. Ci. Inst. Nac. Tecnol. Agropecu. 3: 53-57.

DURANT, S. M. \& et al. 2012. Forgotten biodiversity in desert ecosystems. Science 336: 1379-1380.

FABRIS, H.A. 1983. Gentianaceae. In: A.L. CABRERA (ed.), Flora de la Provincia de Jujuy, Colecc. Ci. Inst. Nac. Tecnol. Agropecu. 13(8): 55-84.

FAO, 2007. Secuestro de Carbono en tierras áridas. Informe sobre recursos mundiales de suelos. $\mathrm{N}^{\mathrm{o}}$ 102. Organización de las Naciones Unidas para la Agricultura y la Alimentación, Roma.

GALÁN DE MERA, A., HAGEN M.A. \& J.A. VICENTE ORELLANA. 1999. Aerophyte, a new life form in Raunkiaer's classification? J. Veg. Sci. 10: 65-68.

GARCÍA, D. 2011. Efectos biológicos de la fragmentación de hábitats: nuevas aproximaciones para resolver un viejo problema. Ecosistemas 20: 1-10.

GIUSTI, L. 1967. Chenopodiaceae. In: Fl. Buenos Aires, Colecc. Ci. Inst. Nac. Tecnol. Agropecu. 3: 81-127.

HALFFTER, G \& E. EZCURRA. 1992. ¿Qué es la biodiversidad?. In: HALFFTER, G. (Comp.), La diversidad biológica de Iberoamérica I. Acta Zool. Mex. (número especial). pp. 2-24. Xalapa, Veracruz, México.

HOSSEUS, C.C. 1916. En las montañas Riojanas al oeste del nevado de Famatina y en regiones limítrofes de San Juan. An. Soc. Cient. Argent. 82: 11-56.

HOSSEUS, C.C. 1921. Estudios comparativos sobre la vegetación de la Provincia de La Rioja y San Juan. Bol. Acad. Nac. Cienc. Córdoba. 26 (1): 5-160.

HUNZIKER, J.H. 1952. Las comunidades vegetales de la Cordillera de La Rioja. Rev. Inv. Agr. Tomo V: 167-196.

INTA, 2002. Olivo: el cultivo con mayor crecimiento. INTA- informa 123: 2.

JUÁREZ, F.C, 1996. Primulaceae. In: NOVARA, L.J. (Dir), Flora del Valle de Lerma. Aportes Bot. Salta, sér. Flora 4(6): 1-12.

JUÁREZ, F.C, 1997. Gentianaceae. In: NOVARA, L.J. (Dir), Flora del Valle de Lerma. Aportes Bot. Salta, sér. Flora 4(20): 1-24.

KIESLING, R. 1983. Primulaceae. In: CABRERA, A.L. (ed.), Flora de la Provincia de Jujuy, Colecc. Ci. Inst. Nac. Tecnol. Agropecu. 13(8): 17-22. 
KIESLING, R. 1994. Hydnoraceae. In: KIESLING, R. (ed.) Flora de San Juan 1: 69-70. Vázques Mazzini, Buenos Aires.

KIESLING, R. 2003. Tamaricaceae. In: KIESLING, R. (ed.) Flora de San Juan 2: 138. Estudios Sigma. Buenos Aires.

KIESLING, R. \& S. MEGLIOLI. 2003. Cactaceae. In: KIESLING, R. (ed.) Flora de San Juan 2: 161-193. Estudios Sigma. Buenos Aires.

LUCENA, I.D. 1993. Nyctaginaceae. In: NOVARA, L.J. (Dir), Flora del Valle de Lerma. Aportes Bot. Salta, sér. Flora 1(24): 1-29.

LUCENA, I.D., \& et al. 2001. Apiaceae. In: NOVARA, L.J. (Dir.), Flora del Valle de Lerma. Aportes Bot. Salta, sér. Flora 6 (11): 1-60.

MÁRQUEZ, J. MARTINEZ CARRETERO, E., DALMASSO, A., PASTRÁN, A. \& G. ORTIZ. 2005. Las áreas protegidas de la Provincia de San Juán (Argentina) II. La vegetación del Parque Provincial Ischigualasto. Multequina 14: 1-27.

MARTÍNEZ CARRETERO, E. 2009. Flora de Mendoza: Anacardiaceae. Multequina 18: 154-165.

McNEELY, J.A. 2003. Biodiversity in arid regions: value and perceptions. J. Arid Environ. 54:61-70.

MEA, 2005a. Ecosystems and Human well-being: Desertification Synthesis. Millenium Ecosystem Assessment. Island Press, Washington, D.C.

MEA, 2005b. 2005. Ecosystems and human well-being: Synthesis. Millennium Ecosystem Assessment, Island Press, Washington, D.C.

MORELLO, J. 1958. La provincia fitogeográfica del monte. Opera Lilloana 2: 5-155.

MORENO-CALLES, A. I. \& A. CASAS. 2008. Conservación de biodiversidad y sustentabilidad en sistemas agroforestales de zonas áridas del Valle de Tehuacán-Cuicatlán, México. Zonas Áridas 12: 10-25.

MÚlgURA, M.E. 1994. Chenopodiaceae. In: KIESLING, R. (ed), Flora de San Juan I: 86-109. Vázques Mazzini, Buenos Aires.

MÚlgURA, M.E. 1987. Nyctaginaceae. In: TRONCOSO N.S. \& N.M. BACIGALUPO (eds.), Flora ilustrada de Entre Ríos, Colecc. Ci. Inst. Nac. Tecnol. Agropecu. 6(3a): 204-209.

MÚGICA DE LA GUERRA, M., LUCIO FERNÁNDEZ, J.V., MARTÍNEZ ALANDI, C., SASTRE OLMOS, P. \& J.A. ATAURI-MEZQUIDA. 2002. Integración territorial de espacios naturales protegidos y conectividad ecológica en paisajes mediterráneos. Texto bilingue (español-inglés). Consejería de Medio Ambiente, Junta de Andalucía, España.

NOVARA, L.J. \& D.G. GUTIERREZ. 2010. AsteraceaeTribu Heliantheae. In: NOVARA, L.J. (Dir), Flora del Valle de Lerma. Aportes Bot. Salta, sér. Flora 9(6): 1-201.
NOVARA, L.J. 1993. Celastraceae. In: NOVARA, L.J. (Dir), Flora del Valle de Lerma. Aportes Bot. Salta, sér. Flora 2(10): 1-13.

NOY-MEIR, Y. 1973. Desert ecosystems: environments and producers. Annu. Rev. Ecol. Syst. 4:25-51.

PASMA - Pcia. La Rioja. 2001. Suelos. Información Geoambiental Minera de Base. Secretaría de Minería de la Nación Argentina. Disponible en: http://www. mineria.gob.ar/estudios/irn/lrioja/F-63.asp\#m5.

PHALAN, B., BERTZKY, M., BUTCHART, S.H.M., DONALD, P.F., SCHARLEMANN, J.P.W., \& A.J. STATTERSFIELD. 2013. Crop Expansion and Conservation Priorities in Tropical Countries. PLoS ONE 8: 1-13.

RAHN, K. 1979. Plantaginaceae. In: TRONCOSO N.S. \& N.M. BACIGALUPO (eds.) Flora ilustrada de Entre Ríos, Colecc. Ci. Inst. Nac. Tecnol. Agropecu. 5(5): 583-591.

RAUNKIAER, C., 1934. The Life Forms of Plants and Statistical Geography. Ed. Claredon. Oxford.

ROIG, F.A., ROIG-JUÑNENT, S. \& V. CORBALÁN. 2009. Biogeography of the Monte Desert. J. Arid Environ. 73: 164-172.

ROSA, H. \& M. MAMANÍ. 2000. Geomorfología de La Rioja. In: Catálogo de recursos humanos e información relacionada con la temática ambiental en la región andina Argentina. Caracterización general y estudios sistemáticos por Provincia. Disponible en: http:/www.cricyt.edu.ar/ladyot/ catalogo/cdandes/cap17.htm\#inhalt.

SALVADOR, V. 2010. Impacto sobre el hábitat. In: VIGLIZZO E.F. \& E. JOBBÁGY (eds.), Expansión de la Frontera Agropecuaria en Argentina y su Impacto Ecológico-Ambiental. Capítulo 7. Ediciones INTA. pp. 43-51.

SEARLES, P.S., AGÜERO ALCARÁS, M. \& M.C. ROUSSEAUX. 2011. El consumo del agua por el cultivo de olivo (Olea europaea L.) en el noroeste de Argentina: una comparación con la Cuenca Mediterránea. Ecol. Austral 21:15-28.

SIMONETTI, J. 1996. Biodiversidad y su conservación en las zonas áridas y semiáridas de América Latina. In: SIMONETTI, J. (Comp.), Conservación y uso sostenible de la biodiversidad en zonas áridas y semiáridas de América Latina y El Caribe. Estudios de casos. Oficina Regional de la FAO para América Latina y El Caribe. Serie Zonas áridas y Semiáridas $N^{o}$ 8. pp. 151-153.

SOSA, H. 2000. El clima de la Rioja. En: Catálogo de recursos humanos e información relacionada con la temática ambiental en la región andina Argentina. Caracterización general y estudios sistemáticos por Provincia. Disponible en: http://www.cricyt.edu.ar/ ladyot/catalogo/cdandes/cap16.htm\#inhalt.

SUDHAKAR REDDY, C., HARI KRISHNA, P., 


\section{O. Varela et al. - Diversidad de plantas vasculares de Antinaco-Los Colorados}

MEENA, S.L., BHARDWAJ, R. \& K.C. SHARMA. 2011. Composition of Life forms and Biological spectrum along climatic gradient in Rajasthan, India. Int. J. Environ. Sci. 1: 1632-1639.

TOLABA, J.A \& M. FABBRONI 1998. Plantaginaceae. In: NOVARA, L.J. (Dir), Flora del Valle de Lerma. Aportes Bot. Salta, sér. Flora 5(5): 1-37.

TOLABA, J.A. 2006. Chenopodiaceae. In: NOVARA, L.J. (Dir), Flora del Valle de Lerma. Aportes Bot. Salta, sér. Flora 7(18): 1-48.

TROMBULAK, S.C. \& C.A. FRISELL. 2000. Review of ecological effects of roads on terrestrial and aquatic communities. Conser. Biol.14: 233-253.

VAN ROOYEN, M.W., THERON, G. K., \& N. GROBBELAAR. 1990. Life forms and spectra of flora of Namaqualand, South Africa. J. Arid Environ. 19: $133-145$.

VARELA, O. \& G.S. JAIME. 2010. Nota sobre la estructura poblacional de árboles en la llanura de Los Mogotes Colorados, La Rioja- Argentina. Lilloa 47: $118-122$.
VITA SERMAN, F \& C. MATÍAS. 2013. Programa Nacional Frutales: Cadena Olivo. Informe INTA. Disponible en: http://inta.gob.ar/documentos/zonasolivicolas-de-la-argentina-contexto-y-prospectivade-la-cadena-olivos/.

PÉREZ VÁZQUEZ, A. \& C. LANDEROS SÁNCHEZ. 2009. Agricultura y Deterioro Ambiental. Elementos 73: $19-25$.

ZULOAGA, F. O., MORRONE, O., \& M. J. BELGRANO. 2008. Catálogo de Las Plantas Vasculares Del Cono Sur: (Argentina, Sur de Brasil, Chile, Paraguay y Uruguay). Monogr. Syst. Bot. Missouri Bot. Gard. 107 (I-II-III): 1-3348. Versión actualizada disponible en: www.darwin.edu.ar.

Recibido el 24 de enero de 2015, aceptado el 15 de junio de 2015. 
Bol. Soc. Argent. Bot. 50 (3) 2015

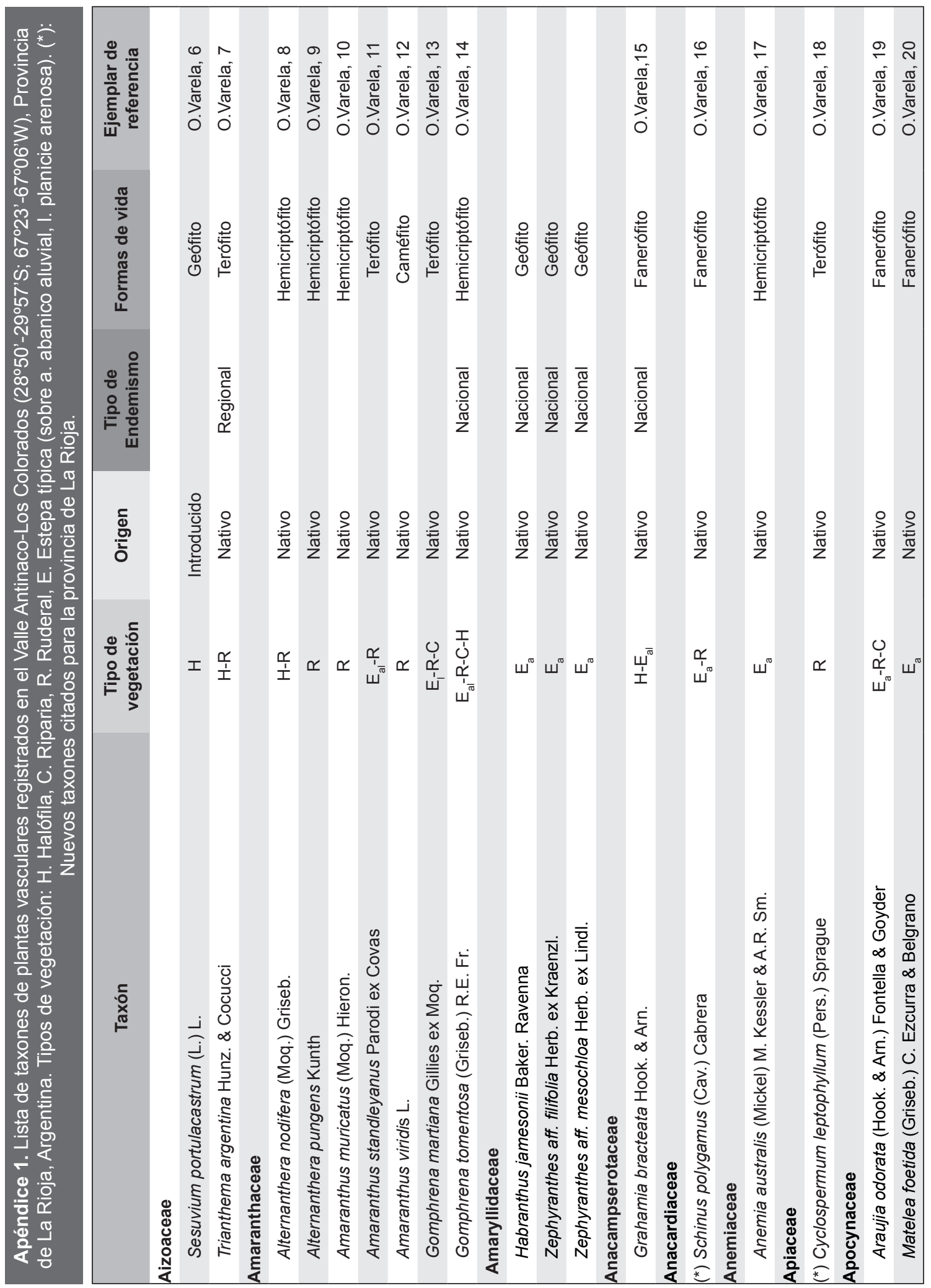




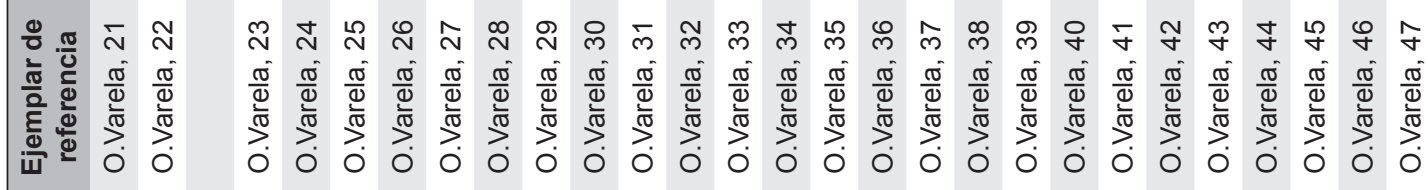

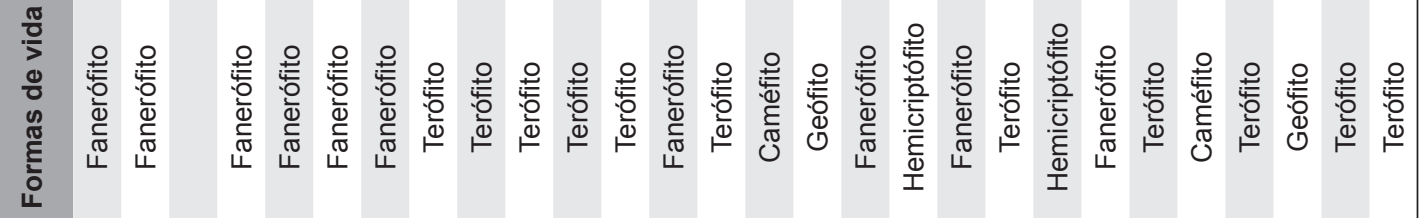

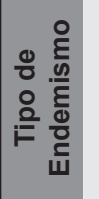
$\frac{\pi}{0}$
$\frac{0}{U}$
$\frac{\pi}{Z}$

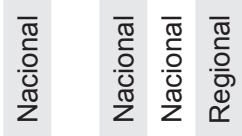

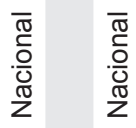

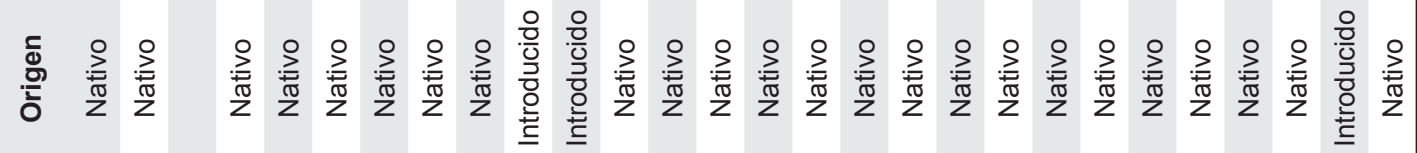

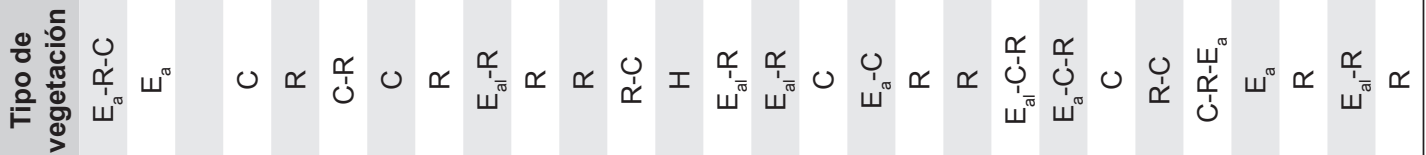

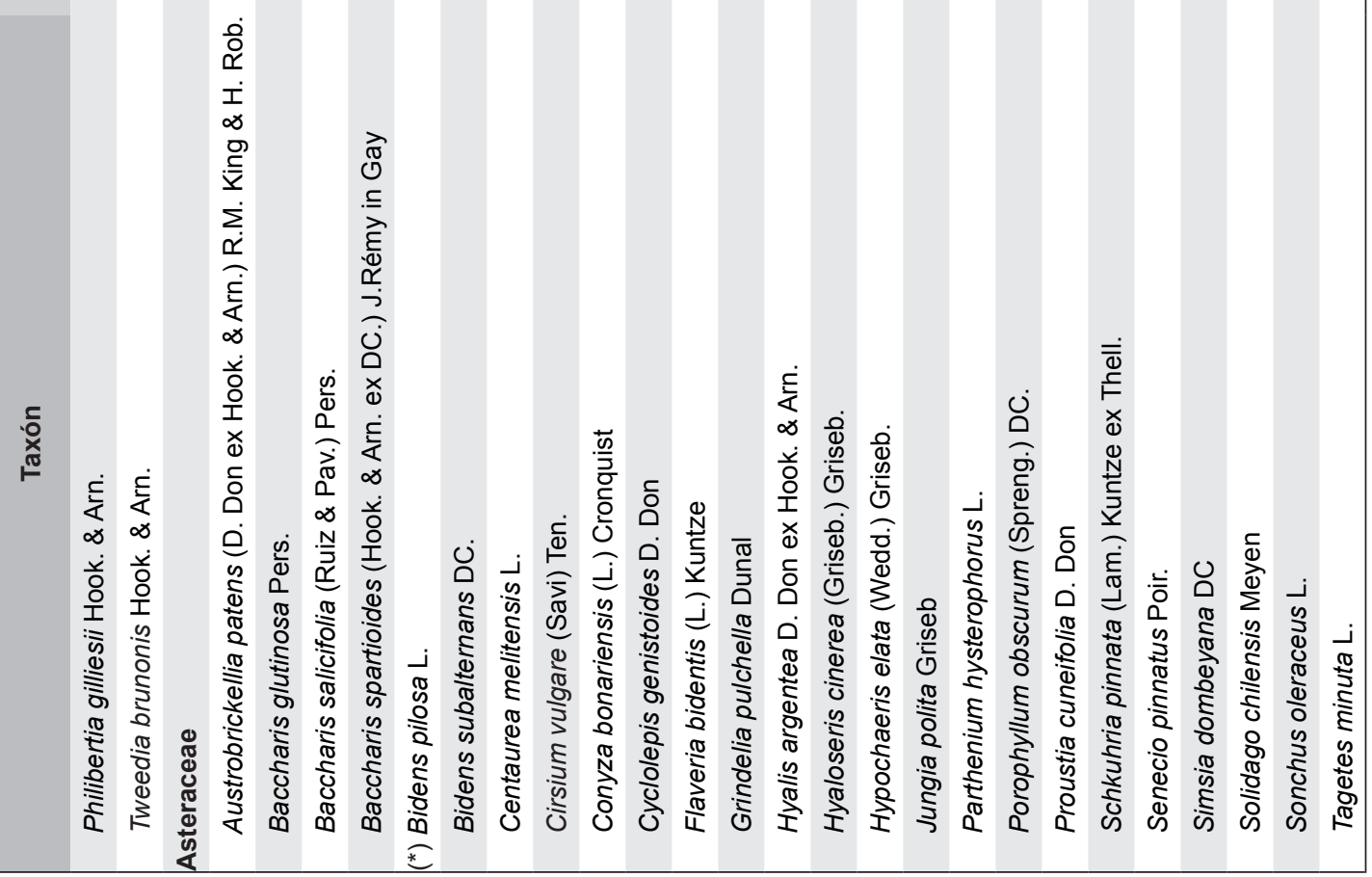




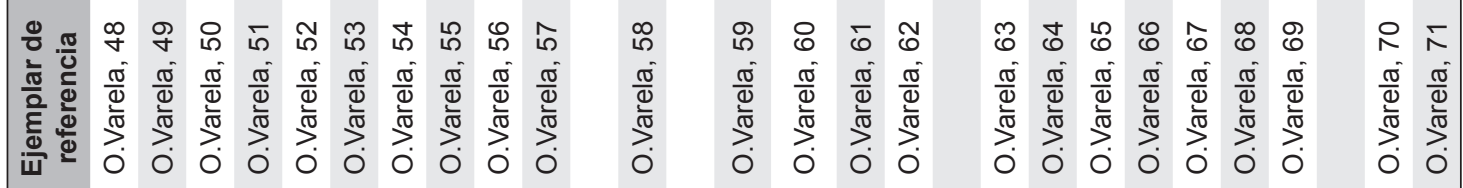

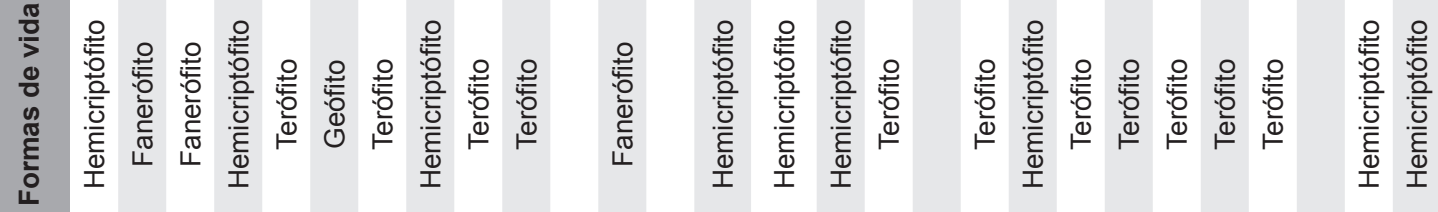

$\frac{\stackrel{0}{\frac{0}{0}}}{\stackrel{\frac{0}{\varepsilon}}{\frac{0}{0}}}$

$\frac{\pi}{2}$
$\frac{0}{0}$
$\frac{0}{Z}$

$\frac{\pi}{2}$
$\frac{1}{2}$
$\frac{0}{2}$
$\frac{0}{2}$

$\frac{\pi}{2}$
$\frac{2}{0}$
$\frac{0}{2}$
$\frac{\pi}{2}$

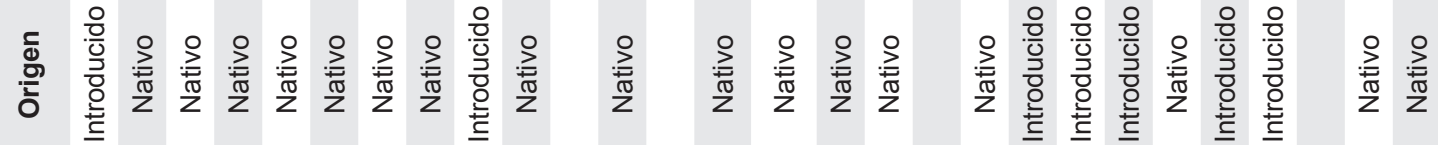

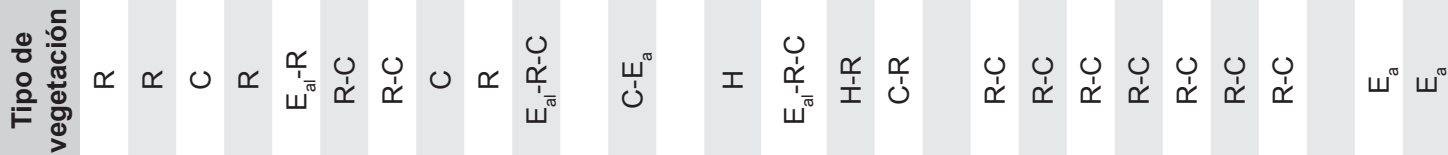

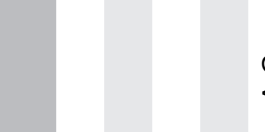

i⿱

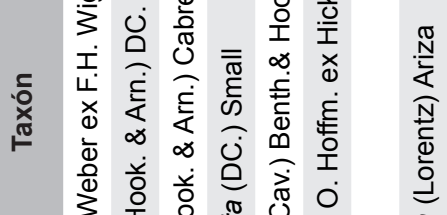

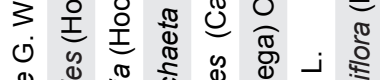

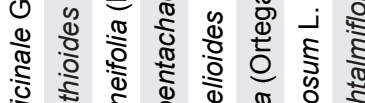

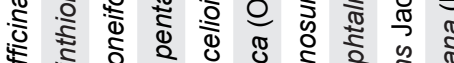

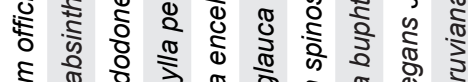

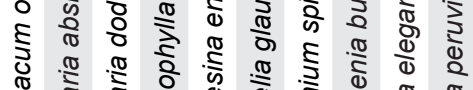

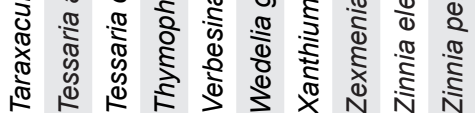

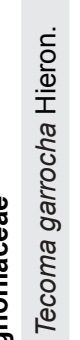

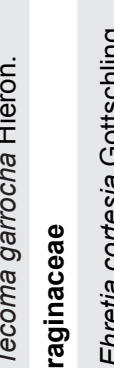

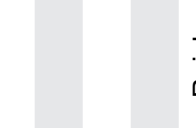

$\frac{\mathscr{c}}{\frac{0}{0}}$

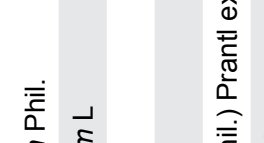

कृ

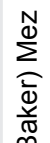

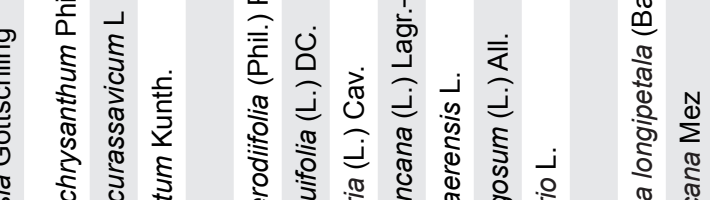

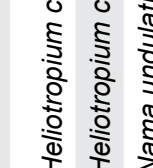
m

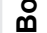



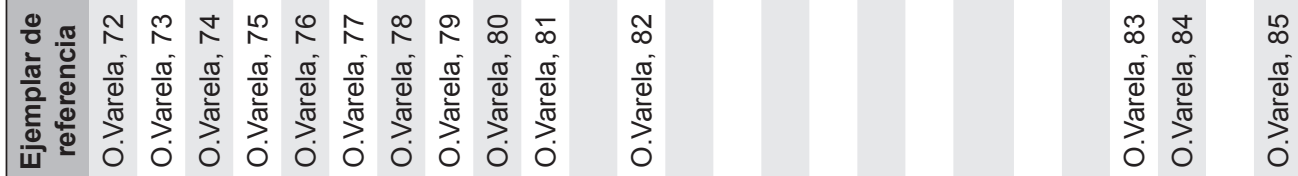

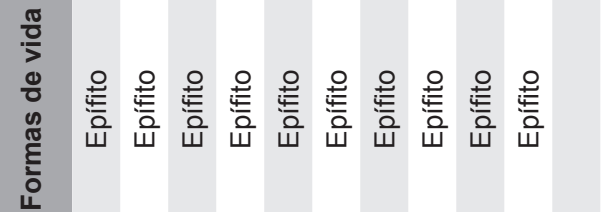

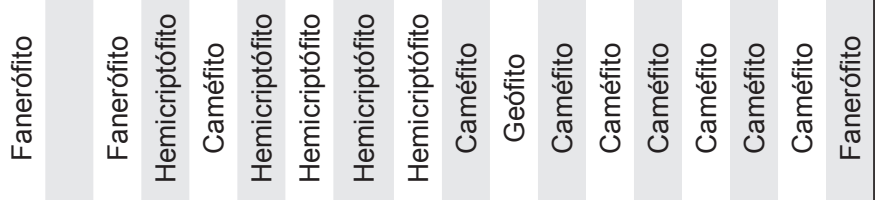

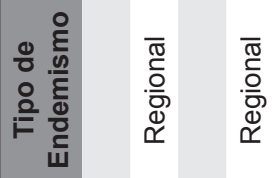

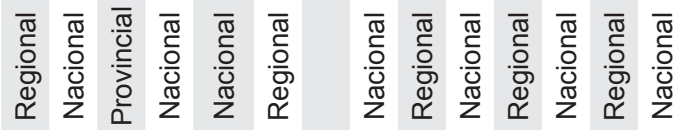

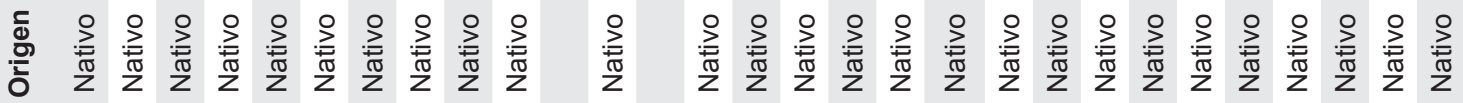

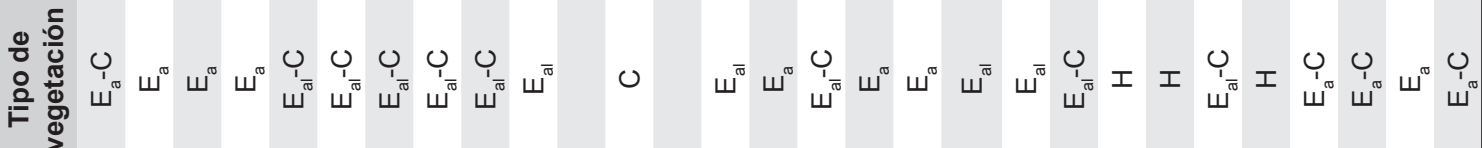

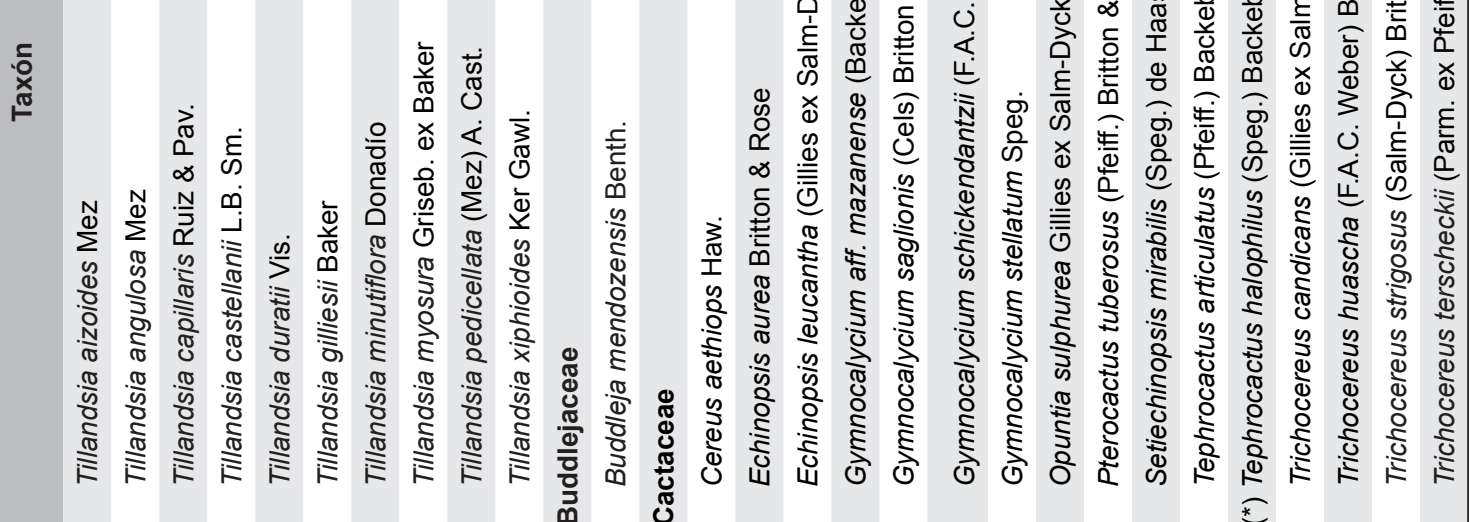




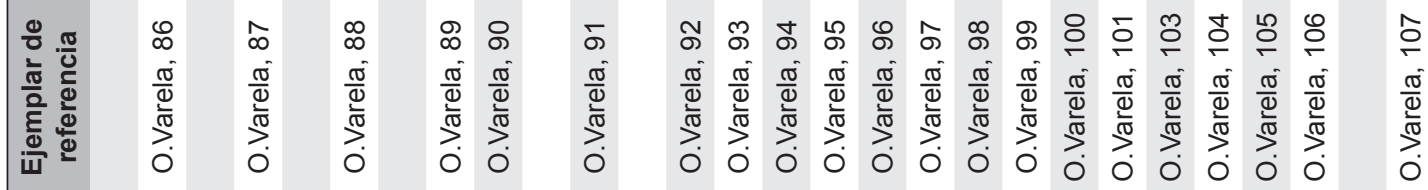

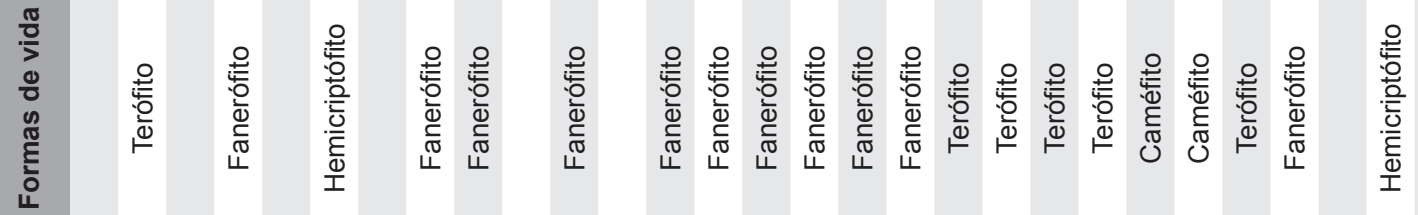

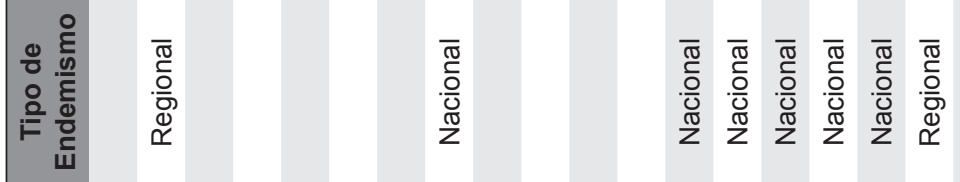

$\overline{0}$
$\frac{0}{0}$
$\frac{0}{2}$

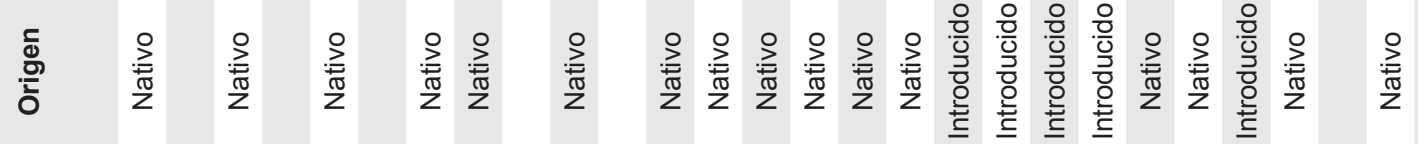

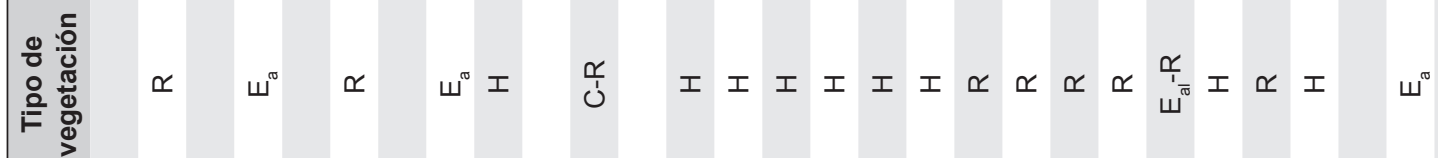

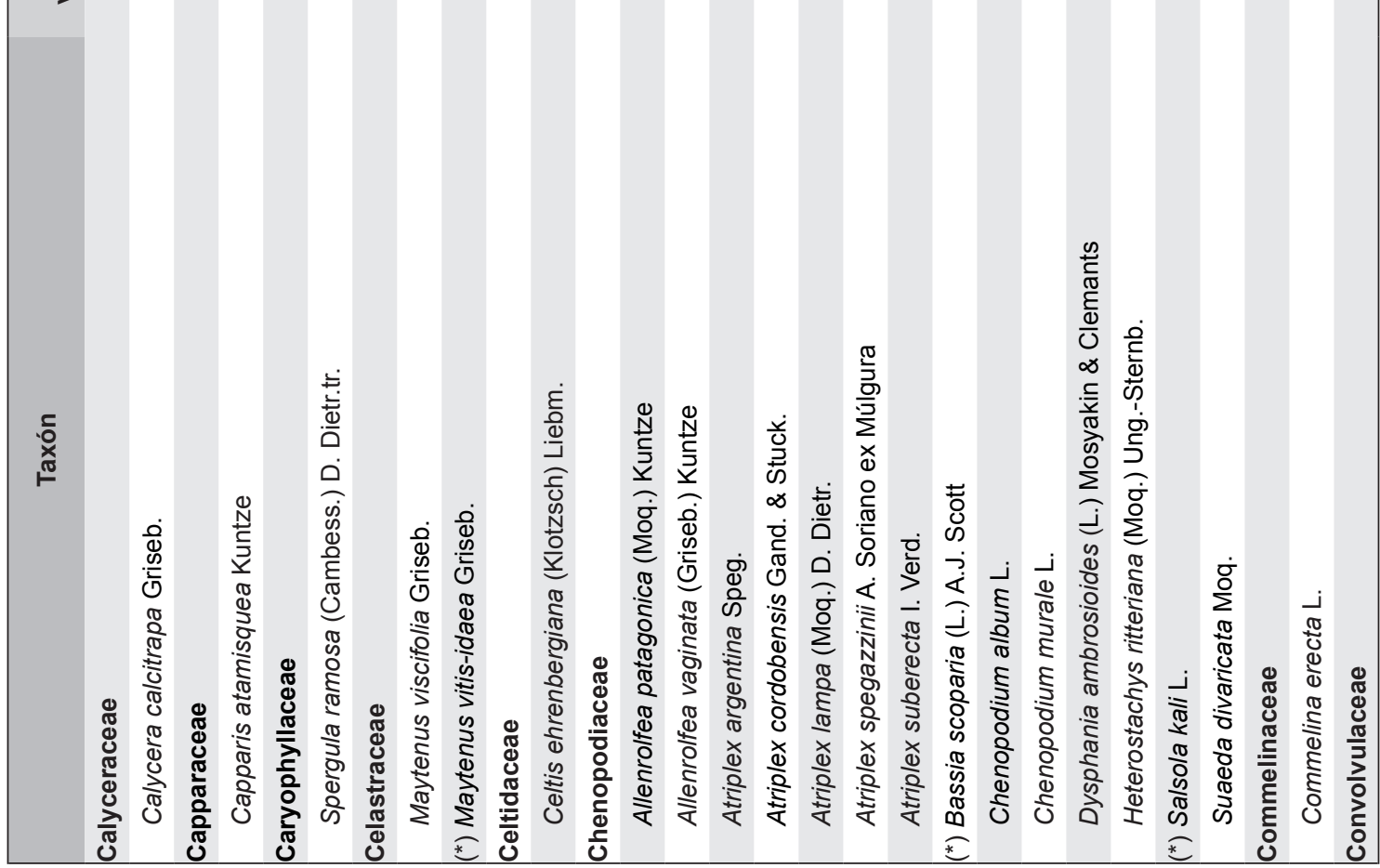




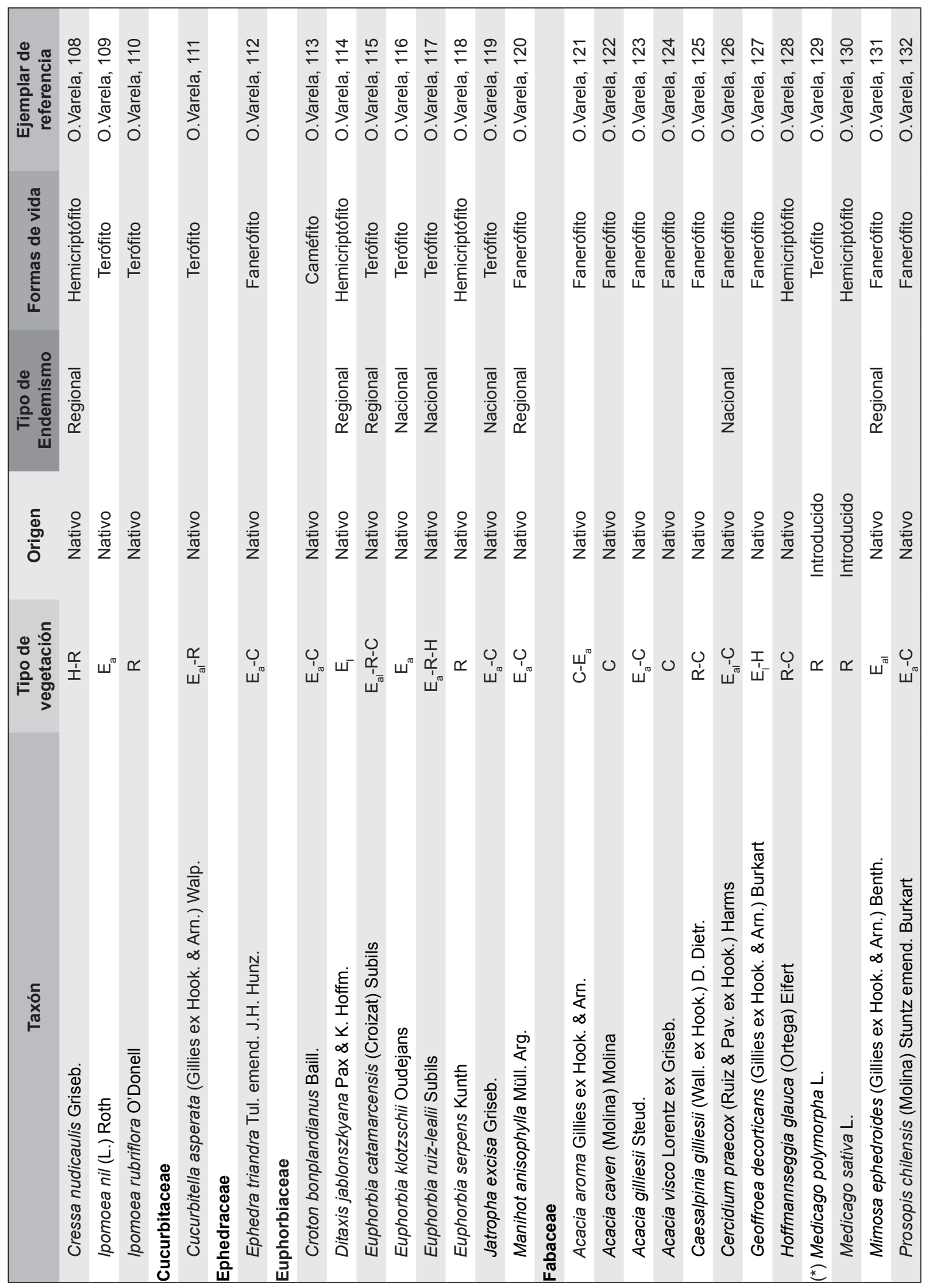




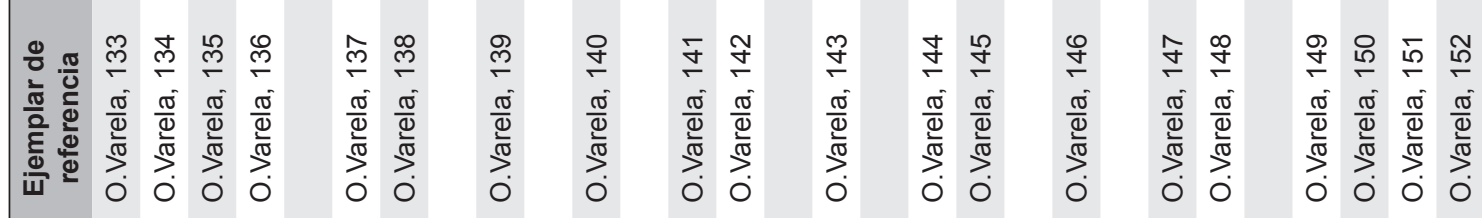

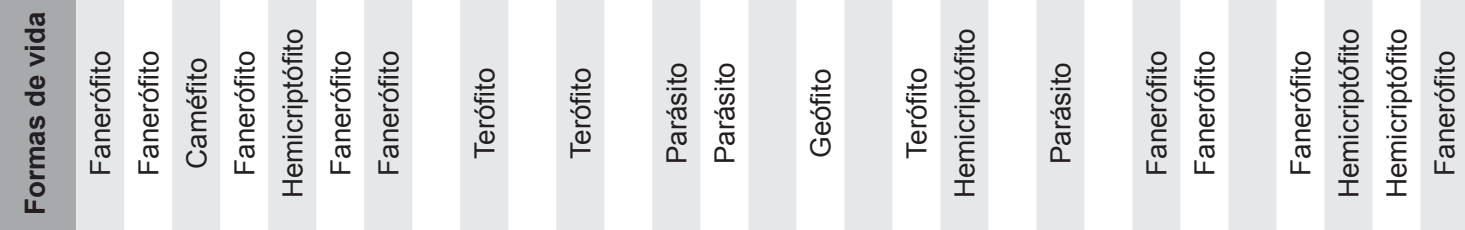

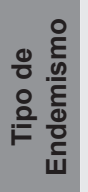
$\begin{array}{ll}\bar{\pi} & \bar{\pi} \\ \overline{\frac{\pi}{0}} & \frac{0}{0} \\ \frac{0}{0} & \frac{0}{0} \\ \frac{\pi}{2} & \frac{\pi}{Z}\end{array}$
$\frac{\pi}{\pi}$
$\frac{0}{0}$
$\frac{\pi}{2}$
$\overline{0}$
$\frac{0}{0}$
$\frac{0}{0}$
$\frac{\pi}{2}$
$\begin{array}{ll}\bar{\pi} & \overline{0} \\ \bar{c} & \frac{0}{0} \\ \frac{0}{U} & \frac{0}{0} \\ \frac{\pi}{z} & \frac{\pi}{z}\end{array}$

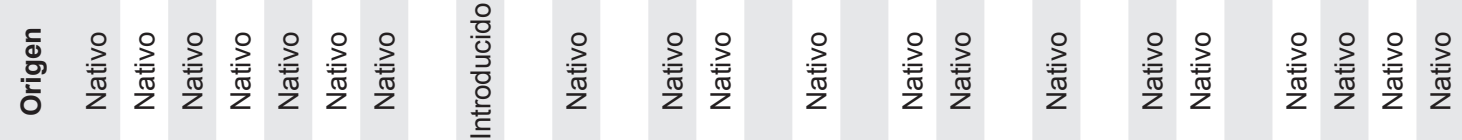

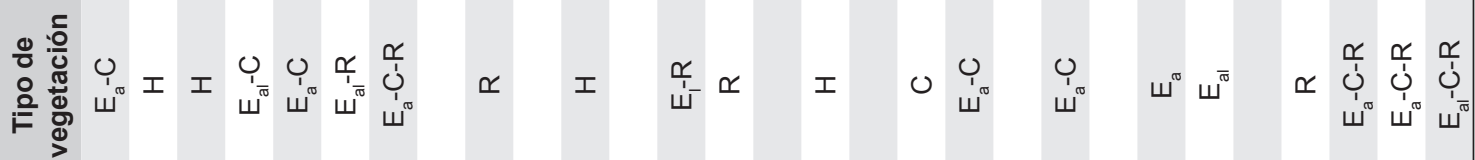
듬
这
亭
๑)

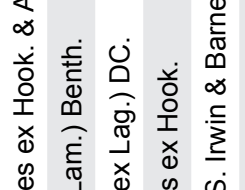

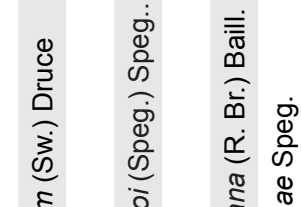

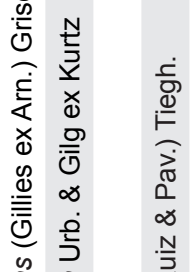

$\frac{\dot{\Phi}}{2}$

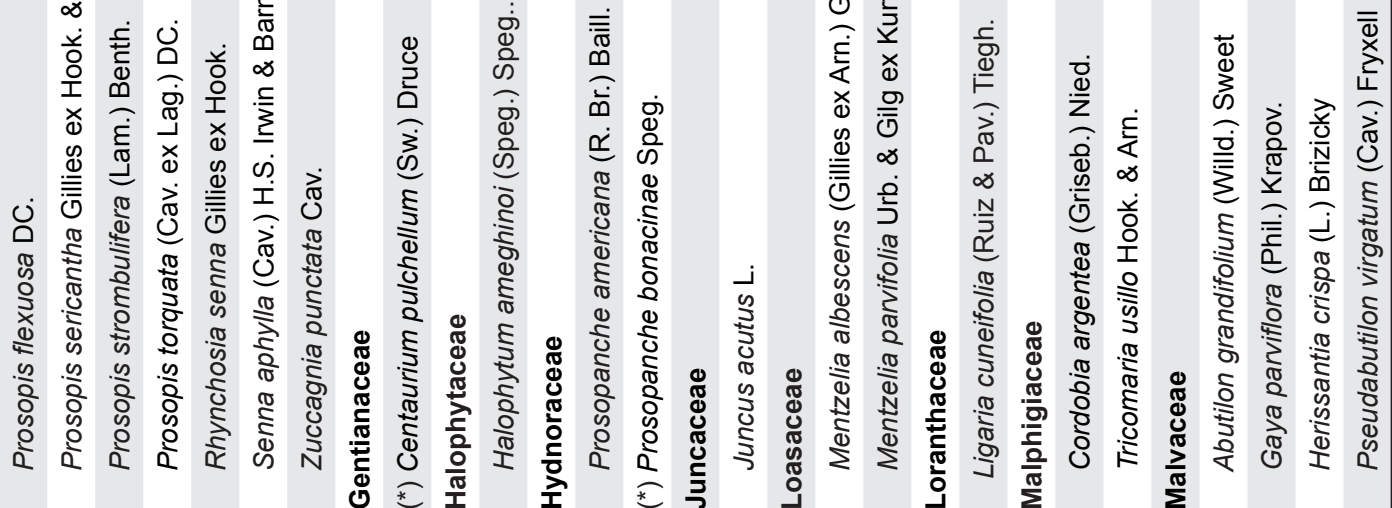




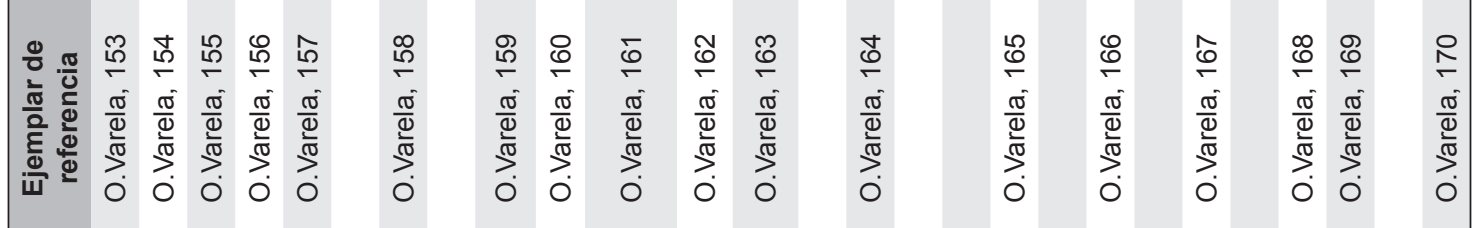

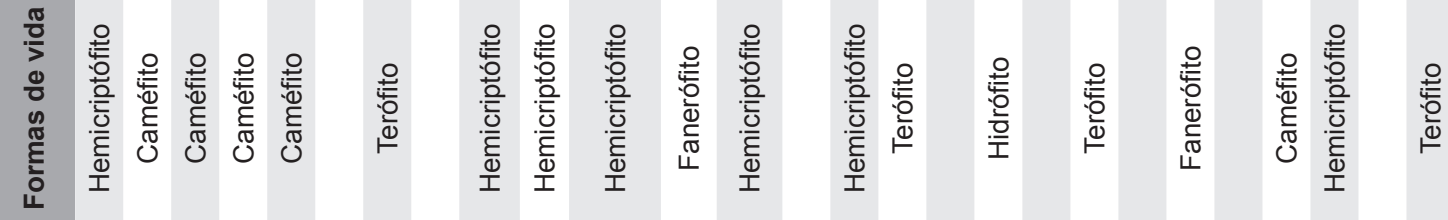

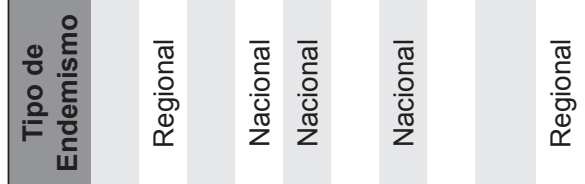

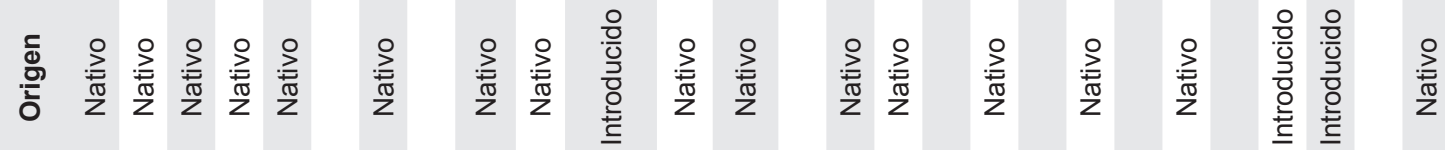

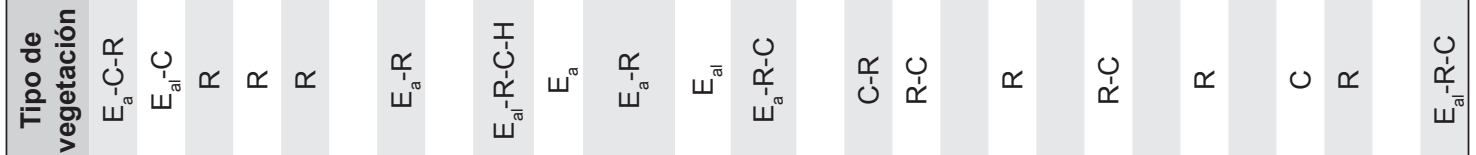

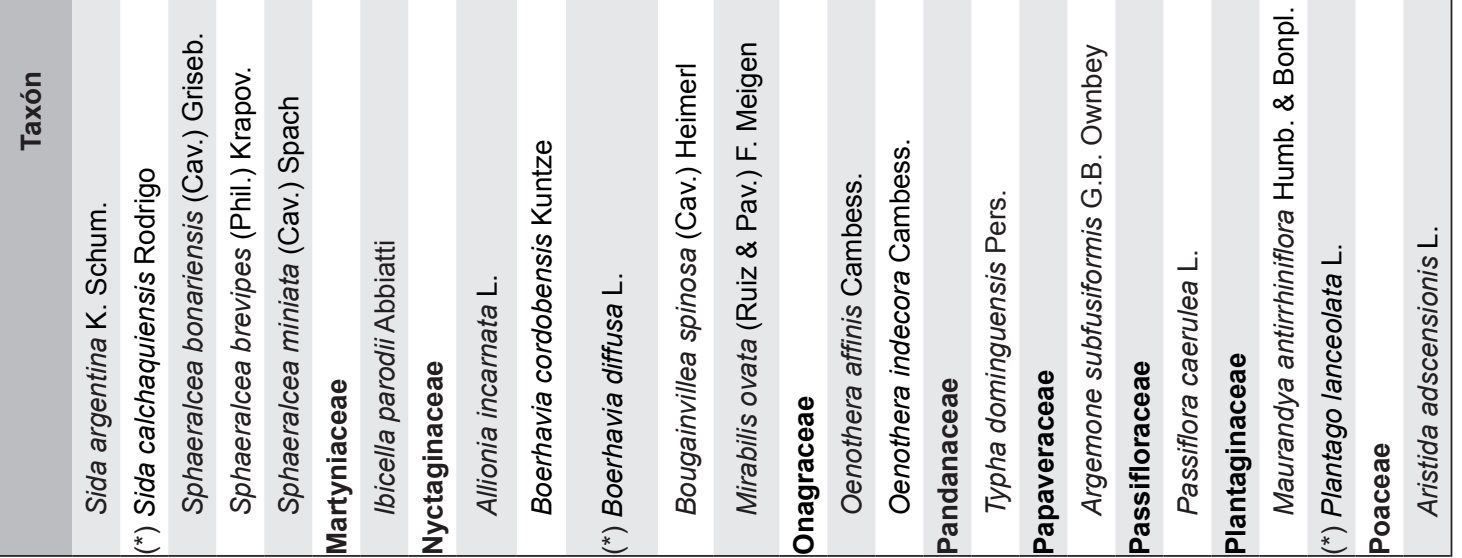




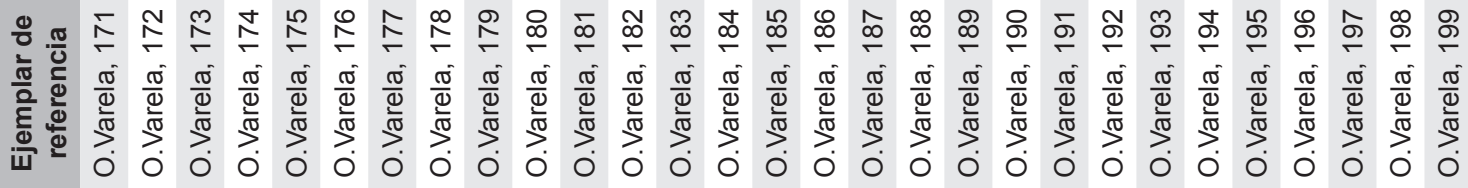

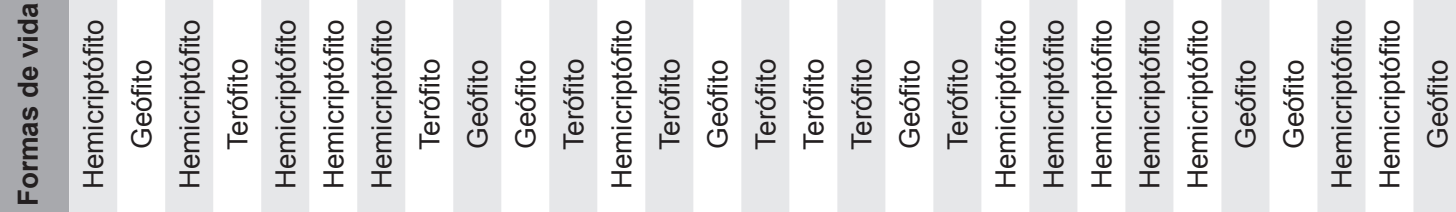

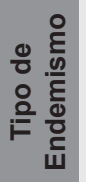

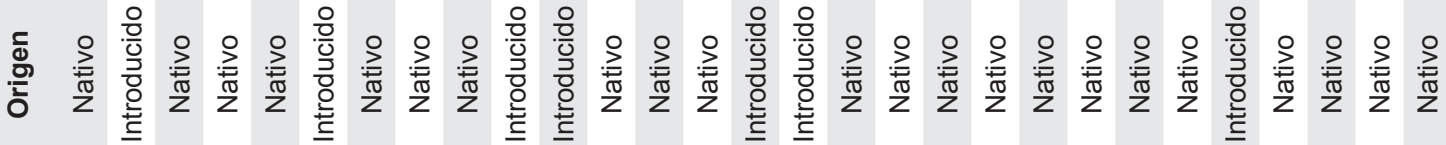

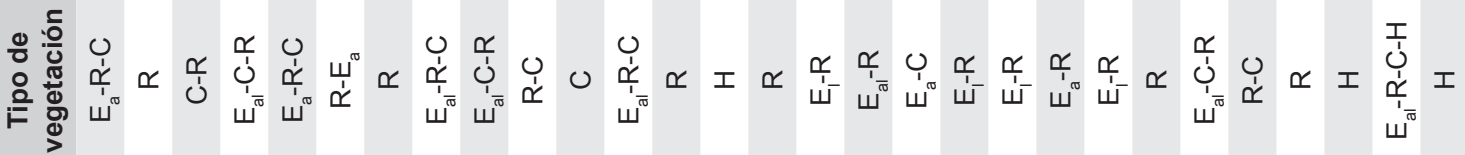

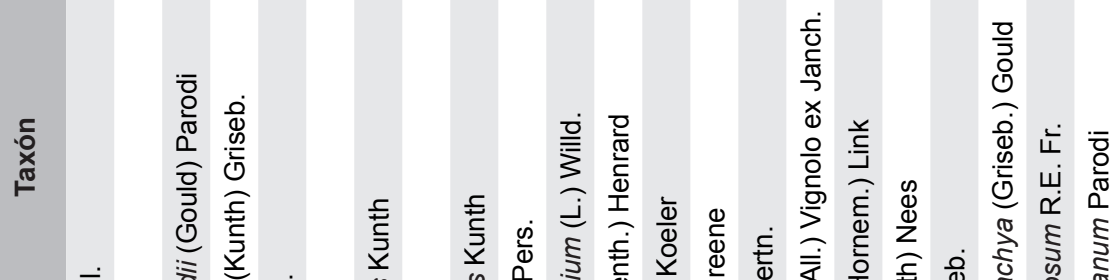

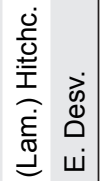

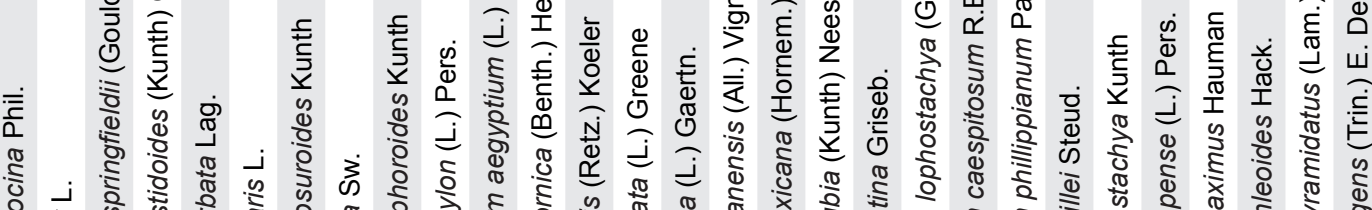

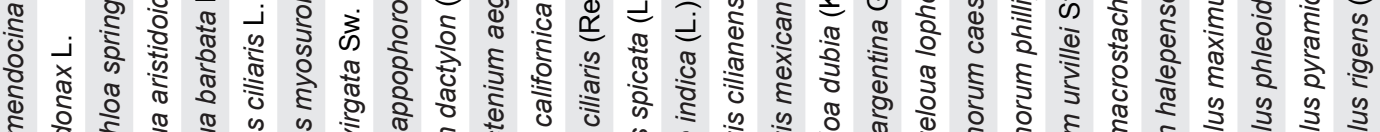

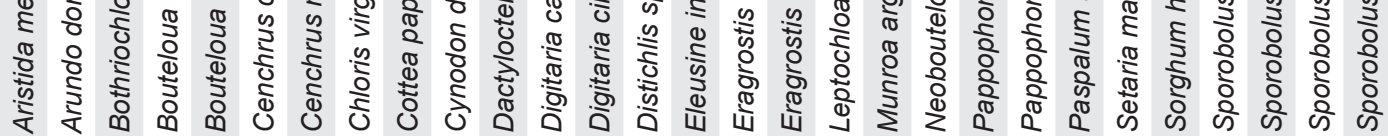




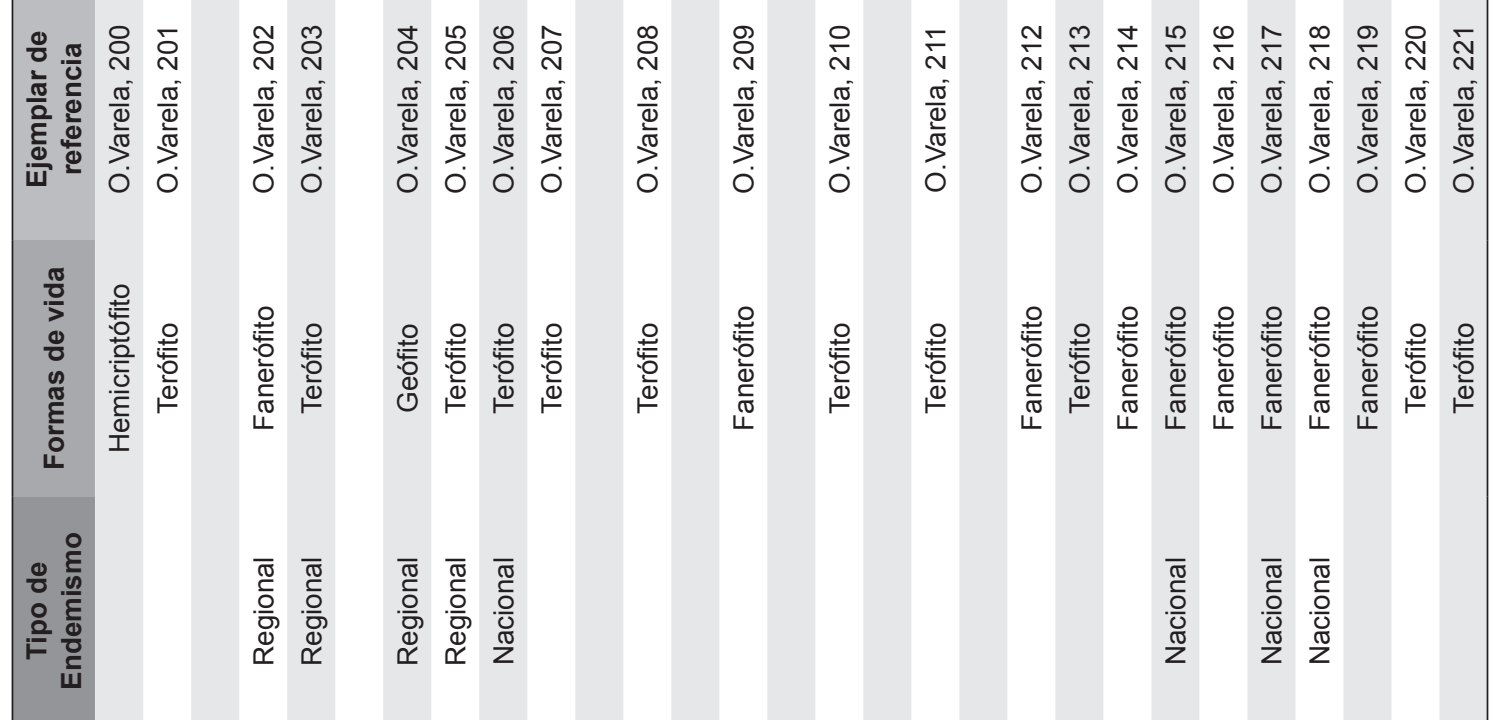

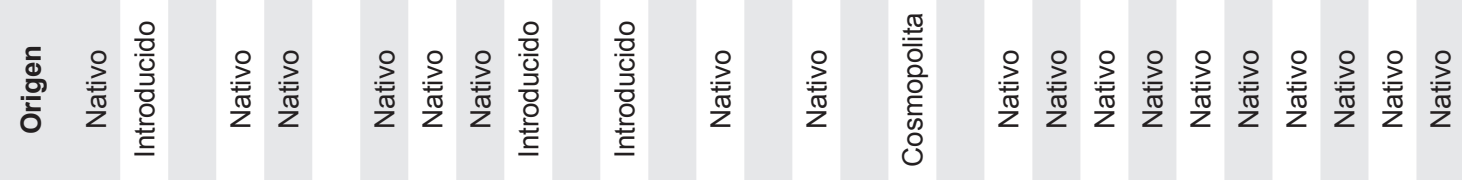

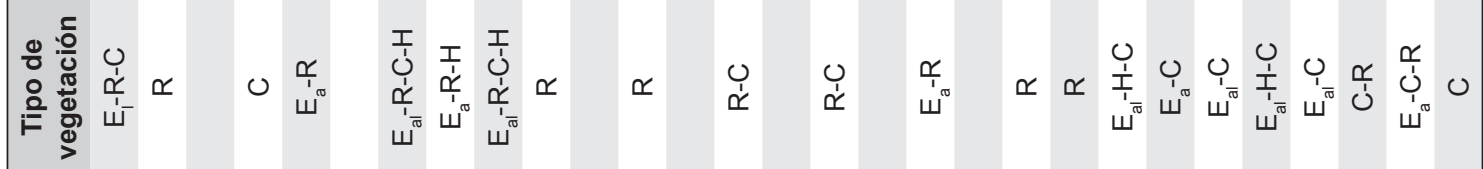

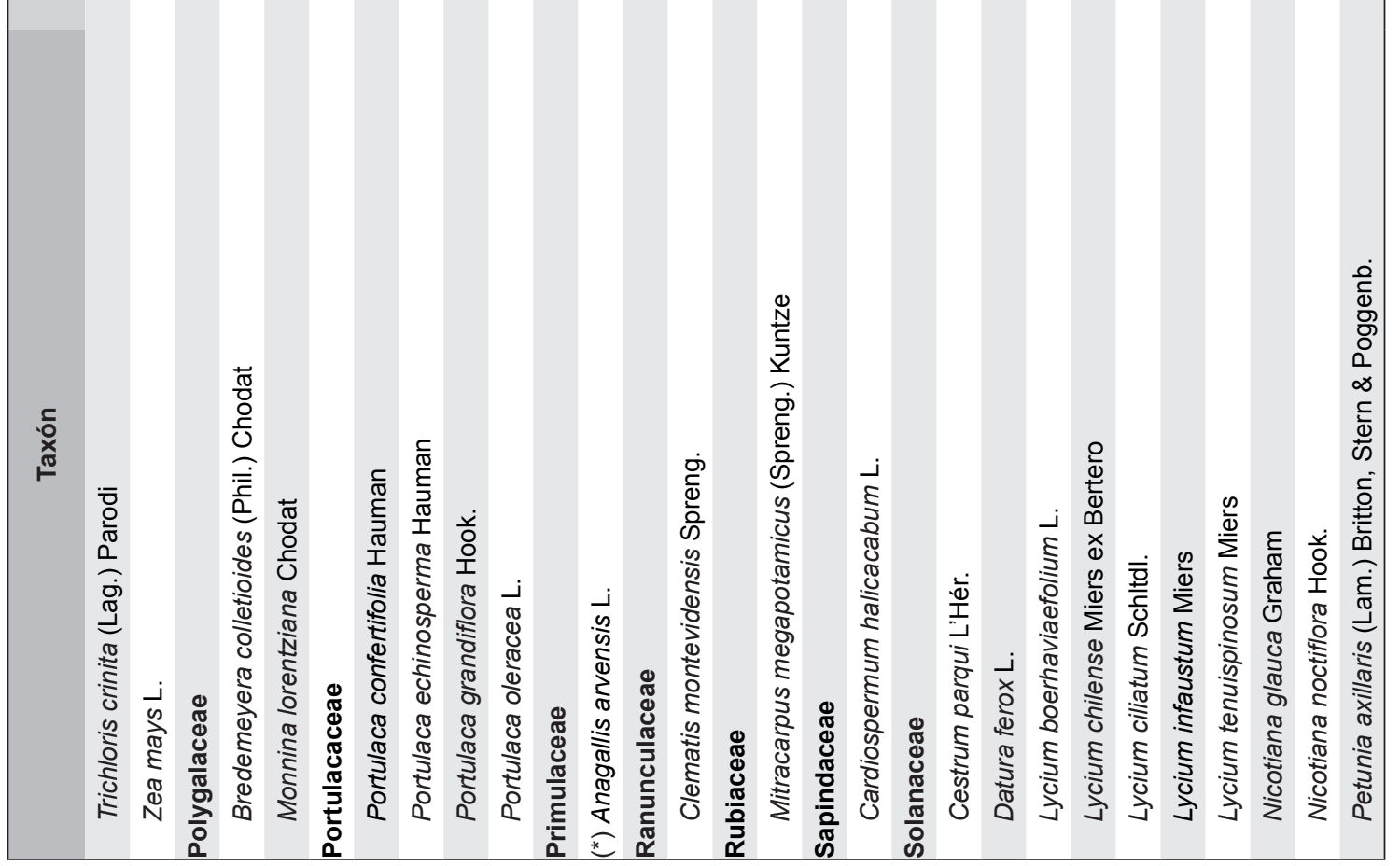




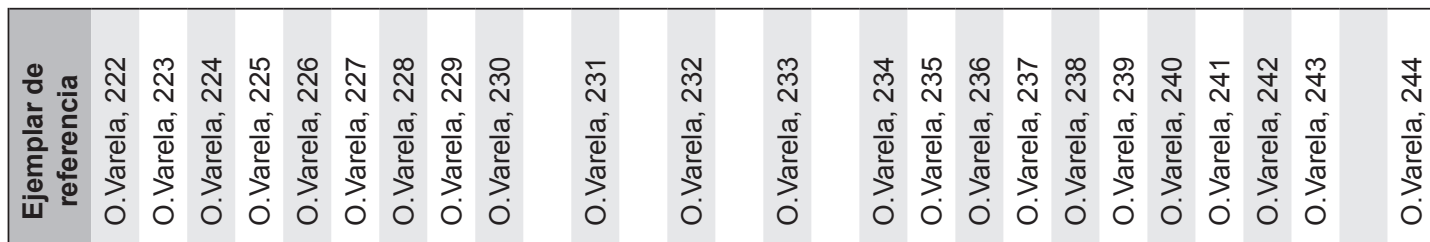

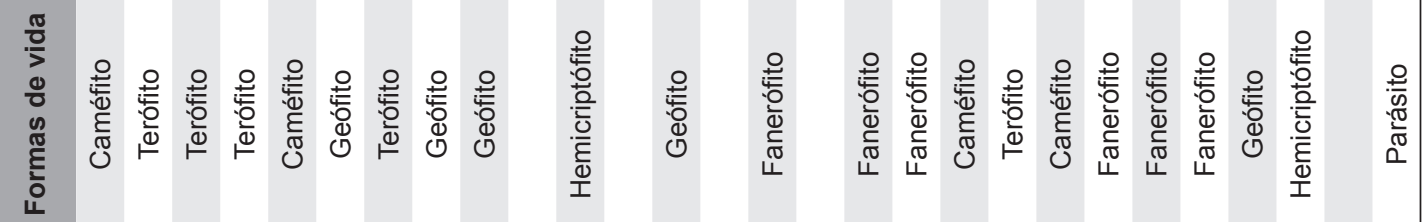

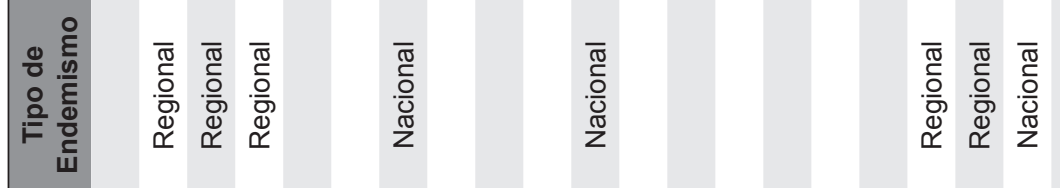

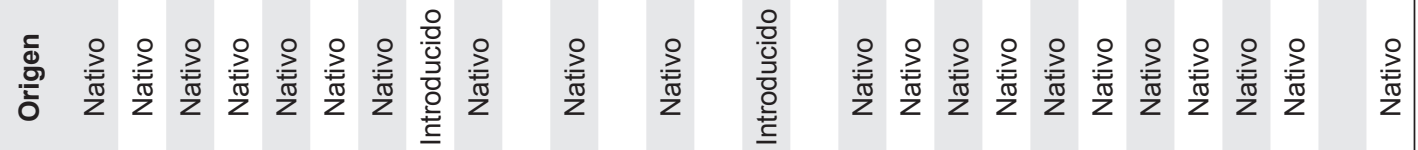

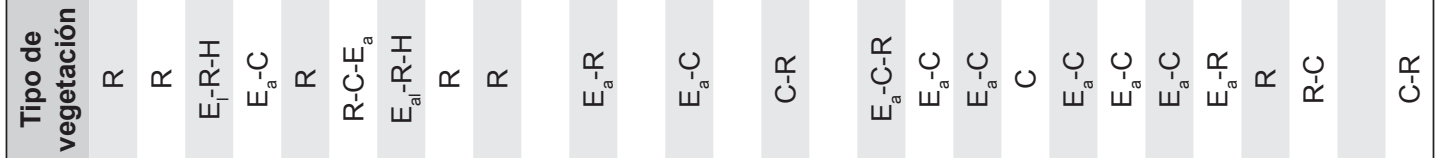

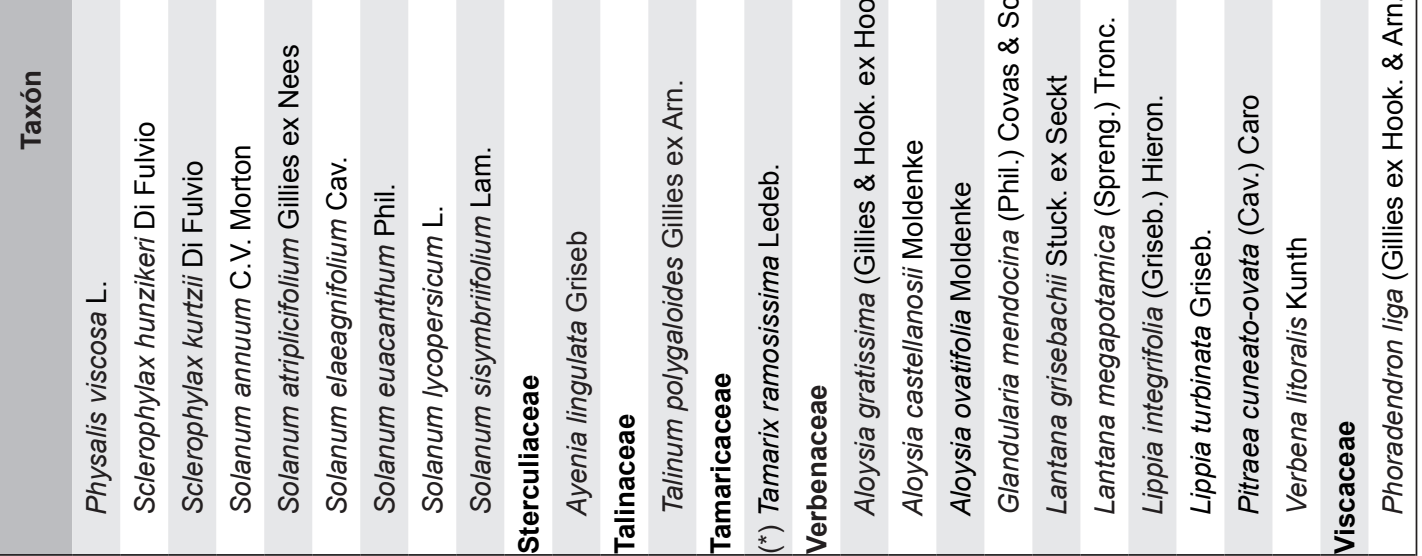


O. Varela et al. - Diversidad de plantas vasculares de Antinaco-Los Colorados

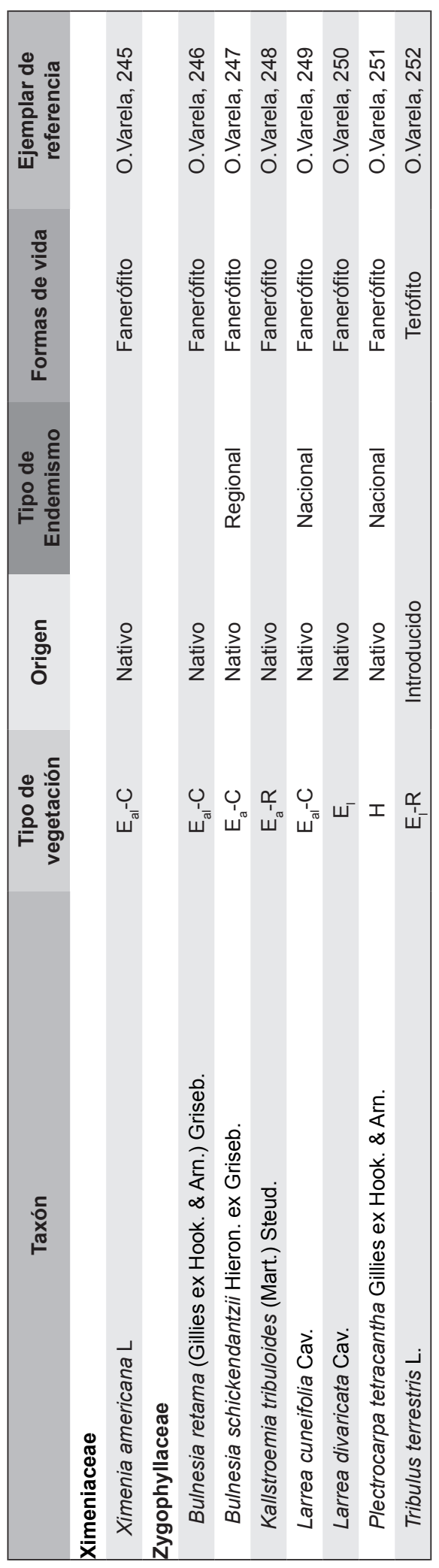


